Journ. Statist. Phys. 88 (1997), 269-305

\title{
Correlations between Zeros of a Random Polynomial
}

\author{
Pavel Bleher ${ }^{1}$ and Xiaojun $\mathrm{Di}^{1}$
}

\author{
Revised version
}

\begin{abstract}
We obtain exact analytical expressions for correlations between real zeros of the Kac random polynomial. We show that the zeros in the interval $(-1,1)$ are asymptotically independent of the zeros outside of this interval, and that the straightened zeros have the same limit translation invariant correlations. Then we calculate the correlations between the straightened zeros of the $\mathrm{SO}(2)$ random polynomial.
\end{abstract}

Key words: Real random polynomials; correlations between zeros; scaling limit; determinants of block matrices.

${ }^{1}$ Department of Mathematical Sciences, Indiana University - Purdue University at Indianapolis, 402 N. Blackford Street, Indianapolis, IN 46202, USA.

E-mail: bleher@math.iupui.edu,xdi@math.iupui.edu. 


\section{Introduction}

Let $f_{n}(t)$ be a real random polynomial of degree $n$,

$$
f_{n}(t)=c_{0}+c_{1} t+\cdots+c_{n} t^{n},
$$

where $c_{0}, c_{1}, \ldots, c_{n}$ are independent real random variables. Distribution of zeros for various classes of random polynomials is studied in the classical papers by Bloch and Polya [BP], Littlewood and Offord [LO], Erdös and Offord [EO], Erdös and Turán [ET], and Kac $[\mathrm{K} 1-\mathrm{K} 3]$. We will assume that the coefficients $c_{0}, c_{1}, \ldots, c_{n}$ are normally distributed with

$$
\mathrm{E} c_{j}=0, \quad \mathrm{E} c_{j}^{2}=\sigma_{j}^{2} .
$$

In the case when

$$
\sigma_{j}^{2}=1,
$$

$f_{n}(t)$ is the Kac random polynomial. Another interesting case is when

$$
\sigma_{j}^{2}=\left(\begin{array}{c}
n \\
j
\end{array}\right) .
$$

As is pointed out by Edelman and Kostlan [EK], "this particular random polynomial is probably the more natural definition of a random polynomial". We call this polynomial the $\mathrm{SO}(2)$ random polynomial because its $m$-point joint probability distribution of zeros is $\mathrm{SO}(2)$-invariant for all $m$ (see section 5 below). The $\mathrm{SO}(2)$ random polynomial can be viewed as the Majorana spin state [Maj] with real random coefficients, and it models a chaotic spin wavefunction in the Majorana representation. See the papers by Leboeuf [Leb1, Leb2], Leboeuf and Shukla [LS], Bogomolny, Bohigas, and Leboeuf [BBL2], and Hannay [Han], where the SU(2) and some other random polynomials are introduced and studied, that represent the Majorana spin states with complex random coefficients.

Let $\left\{\tau_{1}, \ldots, \tau_{k}\right\}$ be the set of real zeros of $f_{n}(t)$. Consider the distribution function of the real zeros,

$$
P_{n}(t)=\mathrm{E} \#\left\{j: \tau_{j} \leq t\right\},
$$

where the mathematical expectation is taken with respect to the joint distribution of the coefficients $c_{0}, \ldots, c_{n}$. Let

$$
p_{n}(t)=P_{n}^{\prime}(t)
$$

be the density function. By the Kac formula (see, e.g., [K3]),

$$
p_{n}(t)=\frac{\sqrt{A_{n}(t) C_{n}(t)-B_{n}^{2}(t)}}{\pi A_{n}(t)} .
$$


where

$$
\begin{aligned}
& A_{n}(t)=\sum_{j=0}^{n} \sigma_{j}^{2} t^{2 j}, \\
& B_{n}(t)=\sum_{j=1}^{n} j \sigma_{j}^{2} t^{2 j-1}=\frac{A_{n}^{\prime}(t)}{2}, \\
& C_{n}(t)=\sum_{j=1}^{n} j^{2} \sigma_{j}^{2} t^{2 j-2}=\frac{A_{n}^{\prime \prime}(t)}{4}+\frac{A_{n}^{\prime}(t)}{4 t} .
\end{aligned}
$$

The derivation of (1.3) by Kac is rather complex. A short proof of (1.3) is given in the paper [EK] by Edelman and Kostlan. See also the papers by Hannay [Han] and Mesincescu, Bessis, Fournier, Mantica, and Aaron [M-A], and section 2 below. The formula (1.3) implies that for the Kac random polynomial,

$$
\lim _{n \rightarrow \infty} p_{n}(t)=p(t)=\frac{1}{\pi\left|1-t^{2}\right|}, \quad t \neq \pm 1
$$

and

$$
p_{n}( \pm 1)=\frac{1}{\pi}\left[\frac{n(n+2)}{12}\right]^{1 / 2}
$$

(see $[\mathrm{K} 3],[\mathrm{BS}]$, and $[\mathrm{EK}]$ ). The limiting density $p(t)$ is not integrable at \pm 1 , and this means that the zeros are mostly located near \pm 1 . Observe, in addition, that $p_{n}(t)$ is an even function of $t$, and the distribution $p_{n}(t) d t$ is invariant with respect to the transformation $t \rightarrow 1 / t$. Kac $[\mathrm{K} 1]$ proves that the expected number of real zeros has the asymptotics

$$
N_{n}=\int_{-\infty}^{\infty} p_{n}(t) d t=(2 / \pi) \log n+O(1)
$$

Kac [K2], Erdös and Offord [EO], Stevens [Ste], Ibragimov and Maslova [IM], Logan and Shepp [LS], Edelman and Kostlan [EK], and others extend this asymptotics to various classes of the random coefficients $\left\{c_{j}\right\}$. Maslova [Mas1] evaluates the variance of the number of real zeros as

$$
\operatorname{Var} \#\left\{j: f_{n}\left(\tau_{j}\right)=0\right\}=\frac{4}{\pi}\left(1-\frac{2}{\pi}\right) \ln n(1+o(1)), \quad n \rightarrow \infty,
$$

and she proves the central limit theorem for the number of real zeros (see [Mas2]), for a class of distributions of the random coefficients $\left\{c_{j}\right\}$.

In this paper we are interested in correlations between the zeros $\tau_{j}$ of the Kac random polynomial. Let us consider first the zeros in the interval $(-1,1)$. Define straightening of $\tau_{j}$ as

$$
\zeta_{j}=P\left(\tau_{j}\right), \quad P(t)=\int_{0}^{t} p(u) d u
$$


In the limit when $n \rightarrow \infty$, the straightened zeros $\zeta_{j}$ are uniformly distributed on the real line, so that

$$
\lim _{n \rightarrow \infty} \mathrm{E} \#\left\{j: a<\zeta_{j} \leq b\right\}=b-a .
$$

From (1.5) we get that

$$
P(t)=\int_{0}^{t} \frac{d u}{\pi\left(1-u^{2}\right)}=\frac{1}{2 \pi} \ln \left|\frac{1+t}{1-t}\right|=\frac{1}{\pi} \operatorname{artanh} t
$$

hence

$$
\zeta_{j}=\frac{1}{\pi} \operatorname{artanh} \tau_{j}
$$

Let $p_{m n}\left(s_{1}, \ldots, s_{m}\right)$ be the joint probability distribution density of the straightened zeros $\zeta_{j}$,

$$
p_{n m}\left(s_{1}, \ldots, s_{m}\right)=\lim _{\Delta s_{1}, \ldots, \Delta s_{m} \rightarrow 0} \frac{\operatorname{Pr}\left\{\exists \zeta_{j_{1}} \in\left[s_{1}, s_{1}+\Delta s_{1}\right], \ldots, \exists \zeta_{j_{m}} \in\left[s_{m}, s_{m}+\Delta s_{m}\right]\right\}}{\left|\Delta s_{1} \ldots \Delta s_{m}\right|} .
$$

It coincides with the correlation function

$$
k_{n m}\left(s_{1}, \ldots, s_{m}\right)=\lim _{\Delta s_{1}, \ldots, \Delta s_{m} \rightarrow 0} \frac{\mathrm{E}\left[\xi_{n}\left(s_{1}, s_{1}+\Delta s_{1}\right) \ldots \xi_{n}\left(s_{m}, s_{m}+\Delta s_{m}\right)\right]}{\left|\Delta s_{1} \ldots \Delta s_{m}\right|} .
$$

where

$$
\xi_{n}(a, b)=\#\left\{j: a<\zeta_{j} \leq b\right\} .
$$

We assume in (1.8) and (1.9) that $s_{i} \neq s_{j}$ for all $i \neq j$. Our aim is to find the limit correlation functions

$$
k_{m}\left(s_{1}, \ldots, s_{m}\right)=\lim _{n \rightarrow \infty} k_{n m}\left(s_{1}, \ldots, s_{m}\right)
$$

We prove the following results.

Theorem 1.1. The limit two-point correlation function $k_{2}\left(s_{1}, s_{2}\right)$ of the straightened zeros $\zeta_{j}=\pi^{-1}$ artanh $\tau_{j}$ of the Kac random polynomial is equal to

$$
k_{2}\left(s_{1}, s_{2}\right)=\tanh ^{2} \pi\left(s_{1}-s_{2}\right)+\frac{\left|\sinh \pi\left(s_{1}-s_{2}\right)\right|}{\cosh ^{2} \pi\left(s_{1}-s_{2}\right)} \arcsin \frac{1}{\cosh \pi\left(s_{1}-s_{2}\right)}
$$

Observe that $k_{2}\left(s_{1}, s_{2}\right)$ depends only on $s_{1}-s_{2}$, and it has the following asymptotics:

$$
\begin{aligned}
& k_{2}\left(s_{1}, s_{2}\right)=\frac{\pi^{2}}{2}\left|s_{1}-s_{2}\right|+O\left(\left|s_{1}-s_{2}\right|^{2}\right), \quad\left|s_{1}-s_{2}\right| \rightarrow 0, \\
& k_{2}\left(s_{1}, s_{2}\right)=1-\frac{16}{3} e^{-4 \pi\left|s_{1}-s_{2}\right|}+O\left(e^{-6 \pi\left|s_{1}-s_{2}\right|}\right), \quad\left|s_{1}-s_{2}\right| \rightarrow \infty
\end{aligned}
$$




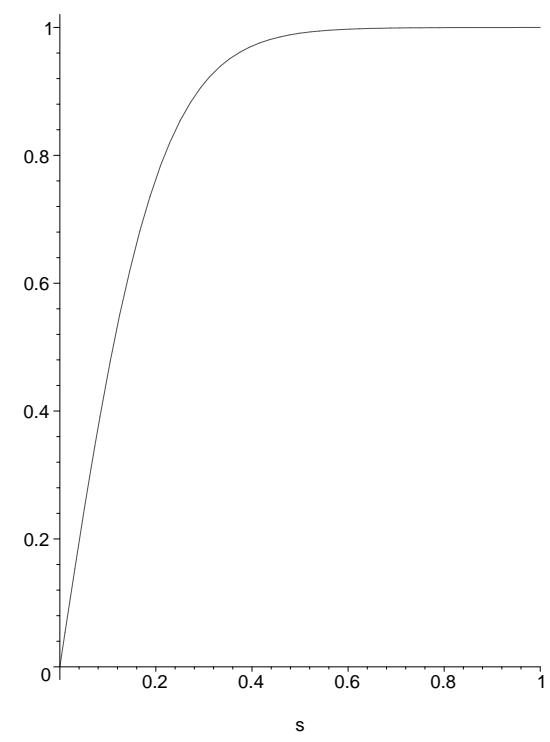

Fig 1: The two-point correlation function of straightened zeros of the Kac random polynomial. The graph of $k_{2}(0, s)$ is given in Fig. 1 .

Theorem 1.2. The limit m-point correlation function $k_{m}\left(s_{1}, \ldots, s_{m}\right)$ of the straightened zeros $\zeta_{j}=\pi^{-1}$ artanh $\tau_{j}$ is equal to

$$
k_{m}\left(s_{1}, \ldots, s_{m}\right)=2^{-m} \int_{-\infty}^{\infty} \ldots \int_{-\infty}^{\infty}\left|y_{1} \ldots y_{m}\right| e^{-\frac{1}{2}\left(Y \Gamma_{m}, Y\right)} d y_{1} \ldots d y_{m},
$$

where $Y=\left(y_{1}, \ldots, y_{m}\right)$ and the matrix $\Gamma_{m}$ is defined as

$$
\Gamma_{m}=\left(\frac{1}{\cosh \pi\left(s_{i}-s_{j}\right)}\right)_{i, j=1}^{m}
$$

In particular, $k_{m}\left(s_{1}, \ldots, s_{m}\right)$ depends only on the differences of $s_{1}, \ldots, s_{m}$, hence it is translation invariant.

The proof of Theorems 1.1 and 1.2 is given in sections 2, 3 and 4 below. It is based on computation of the determinant of some matrices which consist of $2 \times 2$ blocks. This computation is of independent interest. The basic example is the matrix

$$
\Delta_{m}=\left(\begin{array}{cccc}
\Delta_{11} & \Delta_{12} & \cdots & \Delta_{1 m} \\
\Delta_{21} & \Delta_{22} & \cdots & \Delta_{2 m} \\
\ldots & \cdots & \cdots & \cdots \\
\Delta_{m 1} & \Delta_{m 2} & \cdots & \Delta_{m m}
\end{array}\right)
$$

where

$$
\Delta_{i j}=\left(\begin{array}{cc}
\frac{1}{1-t_{i} t_{j}} & \frac{t_{i}}{\left(1-t_{i} t_{j}\right)^{2}} \\
\frac{t_{j}}{\left(1-t_{i} t_{j}\right)^{2}} & \frac{1+t_{i} t_{j}}{\left(1-t_{i} t_{j}\right)^{3}}
\end{array}\right), \quad i, j=1, \ldots, m
$$


We prove in section 4 that

$$
\operatorname{det} \Delta_{m}=\frac{\prod_{1 \leq i<j \leq m}\left(t_{i}-t_{j}\right)^{8}}{\prod_{i=1}^{m}\left(1-t_{i}^{2}\right)^{4} \prod_{1 \leq i<j \leq m}\left(1-t_{i} t_{j}\right)^{8}}
$$

It is interesting to note that determinants of matrices consisting of $2 \times 2$ blocks appear also in the theory of random matrices (see, e.g., [Dys] and [Meh]), statistical physics, and other applications.

Consider now zeros $\tau_{j}$ with $\left|\tau_{j}\right|>1$. Define straightening of $\tau_{j}$ as

$$
\zeta_{j}=P\left(\tau_{j}\right), \quad P(t)=\left\{\begin{array}{l}
\int_{t}^{-\infty} p(u) d u \text { if } t<-1 \\
\int_{t}^{\infty} p(u) d u \text { if } t>1
\end{array}\right.
$$

In the limit when $n \rightarrow \infty$, the straightened zeros $\zeta_{j}$ are uniformly distributed on the real line. From (1.5)

$$
P(t)=\frac{1}{2 \pi} \ln \left|\frac{1+t}{1-t}\right|=\frac{1}{\pi} \operatorname{artanh} t^{-1},
$$

so that

$$
\zeta_{j}=\pi^{-1} \operatorname{artanh} \tau_{j}^{-1}
$$

Denote by $k_{n m}^{\text {out }}\left(s_{1}, \ldots, s_{m}\right)$ the correlation function of the straightened zeros $\zeta_{j}$ with $\left|\tau_{j}\right|>$ 1.

Theorem 1.3.

$$
k_{n m}^{\text {out }}\left(s_{1}, \ldots, s_{m}\right)=k_{n m}\left(s_{1}, \ldots, s_{m}\right) .
$$

In other words, the correlation functions of the straightened zeros outside of the interval $(-1,1)$ coincide with those inside of the interval. Finally, let us consider correlation between zeros inside of the interval $(-1,1)$ and outside of this interval. Let $K_{n m}\left(t_{1}, \ldots, t_{m}\right)$ be the correlation function of the zeros $\tau_{j}$ (without straightening).

Theorem 1.4. Assume that $\left|t_{1}\right|, \ldots,\left|t_{l}\right|<1$ and $\left|t_{l+1}\right|, \ldots,\left|t_{m}\right|>1$. Then the limit

$$
\lim _{n \rightarrow \infty} K_{n m}\left(t_{1}, \ldots, t_{m}\right)=K_{m}\left(t_{1}, \ldots, t_{m}\right)
$$

exists and

$$
K_{m}\left(t_{1}, \ldots, t_{m}\right)=K_{l}\left(t_{1}, \ldots, t_{l}\right) K_{m-l}\left(t_{l+1}, \ldots t_{m}\right)
$$


This means that the zeros inside and outside of the interval $(-1,1)$ are asymptotically independent. Observe that

$$
k_{m}\left(s_{1}, \ldots, s_{m}\right)=\left[\frac{K_{m}\left(t_{1}, \ldots, t_{m}\right)}{p\left(t_{1}\right) \ldots p\left(t_{m}\right)}\right]_{t_{1}=P^{-1}\left(s_{1}\right), \ldots, t_{m}=P^{-1}\left(s_{m}\right)},
$$

provided that either all $\left|t_{j}\right|<1$ or all $\left|t_{j}\right|>1$ (cf. the formula (2.14) below). Proof of Theorems 1.3 and 1.4 is given in the end of section 4.

In sections 5 and 6 we investigate correlation functions of real zeros of the $\mathrm{SO}(2)$ random polynomial.

\section{General Formulae}

Let

$$
f_{n}(t)=\sum_{j=0}^{n} c_{j} t^{j}
$$

be a polynomial whose coefficients $c_{j}$ are random variables with an absolutely continuous joint distribution. Let

$$
\xi_{n}(a, b)=\#\left\{\tau_{k}: a<\tau_{k} \leq b, f_{n}\left(\tau_{k}\right)=0\right\}
$$

be the number of real roots of $f_{n}(t)$ between $a$ and $b$, and let $p_{n}(t)$ be the density of real zeros $t_{k}$ of $f_{n}(t)$, so that

$$
\mathrm{E} \xi_{n}(a, b)=\int_{a}^{b} p_{n}(t) d t
$$

It is not difficult to show that

$$
p_{n}(t)=\int_{-\infty}^{\infty}|y| D_{n}(0, y ; t) d y
$$

where $D_{n}(x, y ; t)$ is a joint distribution density of $f_{n}(t)$ and $f_{n}^{\prime}(t)$,

$$
\operatorname{Pr}\left\{a<f_{n}(t) \leq b ; c<f_{n}^{\prime}(t) \leq d\right\}=\int_{a}^{b} \int_{c}^{d} D_{n}(x, y ; t) d x d y .
$$

Indeed, if $f_{n}^{\prime}(t)=y$ then asymptotically as $\Delta t \rightarrow 0$, the function $f_{n}(t)$ has a zero in the interval $[t, t+\Delta t]$ if $f_{n}(t)$ is in the interval $[0,-y \Delta t]$, and this gives (2.5). Similarly, the $m$-point correlation function $K_{n m}\left(t_{1}, \ldots, t_{m}\right)$ for pairwise different $t_{1}, \ldots, t_{m}$ is equal to

$$
K_{n m}\left(t_{1}, \ldots, t_{m}\right)=\int_{-\infty}^{\infty} \cdots \int_{-\infty}^{\infty}\left|y_{1} \ldots y_{m}\right| D_{n m}\left(0, y_{1}, \ldots, 0, y_{m} ; t_{1}, \ldots, t_{m}\right) d y_{1} \ldots d y_{m}
$$


where $D_{n m}\left(x_{1}, y_{1}, \ldots, x_{m}, y_{m} ; t_{1}, \ldots, t_{m}\right)$ is a joint distribution density of the vector

$$
F_{n}=\left(f_{n}\left(t_{1}\right), f_{n}^{\prime}\left(t_{1}\right), \ldots, f_{n}\left(t_{m}\right), f_{n}^{\prime}\left(t_{m}\right)\right)
$$

so that

$$
\begin{aligned}
\operatorname{Pr} & \left\{a_{1}<f_{n}\left(t_{1}\right) \leq b_{1} ; c_{1}<f_{n}^{\prime}\left(t_{1}\right) \leq d_{1} ; \ldots ; a_{m}<f_{n}\left(t_{m}\right) \leq b_{m} ; c_{m}<f_{n}^{\prime}\left(t_{m}\right) \leq d_{m}\right\} \\
& =\int_{a_{1}}^{b_{1}} \int_{c_{1}}^{d_{1}} \cdots \int_{a_{m}}^{b_{m}} \int_{c_{m}}^{d_{m}} D_{n m}\left(x_{1}, y_{1}, \ldots, x_{m}, y_{m} ; t_{1}, \ldots, t_{m}\right) d x_{1} d y_{1} \ldots d x_{m} d y_{m} .
\end{aligned}
$$

If $\left\{c_{j}\right\}$ are independent random variables with $\operatorname{Var} c_{j}>0$ then the covariance matrix of the vector $F_{n}$ is positive, provided that $n \geq 2 m-1$ (see Appendix $\mathrm{B}$ at the end of the paper). Similar formulae are derived for the correlation functions of complex zeros of random polynomials with complex and real coefficients (see [Han] and [M-A]).

Observe that

$$
\mathrm{E} \prod_{j=1}^{m} \xi_{n}\left(a_{j}, b_{j}\right)=\int_{a_{1}}^{b_{1}} \ldots \int_{a_{m}}^{b_{m}} K_{n m}\left(t_{1}, \ldots, t_{m}\right) d t_{1} \ldots d t_{m}
$$

provided that $\left(a_{1}, b_{1}\right), \ldots,\left(a_{m}, b_{m}\right)$ are pairwise disjoint, and

$$
p_{n}(t)=K_{n 1}(t), \quad \mathrm{E}\left(\xi_{n}(a, b)\right)=\int_{a}^{b} K_{n 1}(t) d t .
$$

For the general case, when $\left(a_{1}, b_{1}\right), \ldots,\left(a_{m}, b_{m}\right)$ may intersect, we have the following extension of $(2.8)$ :

$$
\mathrm{E} \prod_{j=1}^{m} \xi_{n}\left(a_{j}, b_{j}\right)=\sum_{\left(A_{1}, \ldots, A_{l}\right)} \prod_{j=1}^{l}\left(\int_{\bigcap_{i \in A_{j}}\left(a_{i}, b_{i}\right)} d t_{j}\right) K_{n l}\left(t_{1}, \ldots, t_{l}\right),
$$

where the sum is taken over all possible partitions $\left(A_{1}, \ldots, A_{l}\right)$ of $\{1, \ldots, m\}$, such that

$$
\begin{aligned}
& A_{i} \cap A_{j}=\emptyset, \quad i \neq j, \\
& A_{1} \cup \cdots \cup A_{l}=\{1, \ldots, m\}, \\
& \left|A_{i}\right| \geq 1 \quad i=1, \ldots, l .
\end{aligned}
$$

In particular, when $m=2$ we have

$$
\mathrm{E}\left[\xi_{n}\left(a_{1}, b_{1}\right) \xi_{n}\left(a_{2}, b_{2}\right)\right]=\int_{a_{1}}^{b_{1}} \int_{a_{2}}^{b_{2}} K_{n 2}\left(t_{1}, t_{2}\right) d t_{1} d t_{2}
$$


if $\left(a_{1}, b_{1}\right) \cap\left(a_{2}, b_{2}\right)=\emptyset$, and

$$
\mathrm{E}\left[\xi_{n}^{2}(a, b)\right]=\int_{a}^{b} p_{n}(t) d t+\int_{a}^{b} \int_{a}^{b} K_{n 2}\left(t_{1}, t_{2}\right) d t_{1} d t_{2}
$$

From definition (1.9) of the $m$-point correlation function, it follows that the $m$-point correlation function $k_{n m}\left(s_{1}, \ldots, s_{m}\right)$ of the straightened zeros $\zeta_{j}=P\left(\tau_{j}\right)$ is related to the $m$-point correlation function $K_{n m}\left(t_{1}, \ldots, t_{m}\right)$ of the zeros $\tau_{j}$ by the formula

$$
k_{n m}\left(s_{1}, \ldots, s_{m}\right)=\left[\frac{K_{n m}\left(t_{1}, \ldots, t_{m}\right)}{p\left(t_{1}\right) \ldots p\left(t_{m}\right)}\right]_{t_{1}=P^{-1}\left(s_{1}\right), \ldots, t_{m}=P^{-1}\left(s_{m}\right)} .
$$

Assume now that the coefficients $c_{j}$ are independent Gaussian variables with zero mean and the variances $\sigma_{j}^{2}, j=0, \ldots, n$. Then $D_{n 1}(x, y ; t)$ is a Gaussian distribution density with the covariance matrix

$$
\Delta=\left(\begin{array}{cc}
\mathrm{E} f_{n}^{2}(t) & \mathrm{E} f_{n}(t) f_{n}^{\prime}(t) \\
\mathrm{E} f_{n}(t) f_{n}^{\prime}(t) & \mathrm{E}\left(f_{n}^{\prime}(t)\right)^{2}
\end{array}\right)=\left(\begin{array}{cc}
A_{n}(t) & B_{n}(t) \\
B_{n}(t) & C_{n}(t)
\end{array}\right)
$$

where $A_{n}(t), B_{n}(t)$ and $C_{n}(t)$ are defined in (1.4), and from (2.4) we get the Kac formula $(1.3)$.

\section{Two-Point Correlation Function for the Kac Polynomial}

Let $f_{n}(t)=c_{0}+c_{1} t+\cdots+c_{n} t^{n}$ be the Kac polynomial, so that $c_{k}, k=0, \ldots, n$, are real independent Gaussian random variables with

$$
\mathrm{E} c_{k}=0, \quad \mathrm{E} c_{k}^{2}=1
$$

Consider the covariance matrix $\Delta_{n}$ of the Gaussian vector $\left(f_{n}\left(t_{1}\right), f_{n}^{\prime}\left(t_{1}\right), f_{n}\left(t_{2}\right), f_{n}^{\prime}\left(t_{2}\right)\right)$. From (3.1)

$$
\begin{aligned}
& \mathrm{E} f_{n}\left(t_{1}\right) f_{n}\left(t_{2}\right)=\sum_{k=0}^{n}\left(t_{1} t_{2}\right)^{k}=\frac{1-\left(t_{1} t_{2}\right)^{n+1}}{1-t_{1} t_{2}}, \\
& \mathrm{E} f_{n}^{\prime}\left(t_{1}\right) f_{n}\left(t_{2}\right)=\frac{\partial}{\partial t_{1}}\left[\frac{1-\left(t_{1} t_{2}\right)^{n+1}}{1-t_{1} t_{2}}\right], \\
& \mathrm{E} f_{n}^{\prime}\left(t_{1}\right) f_{n}^{\prime}\left(t_{2}\right)=\frac{\partial^{2}}{\partial t_{1} \partial t_{2}}\left[\frac{1-\left(t_{1} t_{2}\right)^{n+1}}{1-t_{1} t_{2}}\right] .
\end{aligned}
$$

Assume that $\left|t_{1}\right|,\left|t_{2}\right|<1$. Then from (3.2) we obtain that

$$
\lim _{n \rightarrow \infty} \Delta_{n}=\Delta
$$


with

$$
\Delta=\left(\begin{array}{cccc}
\frac{1}{1-t_{1}{ }^{2}} & \frac{t_{1}}{\left(1-t_{1}\right)^{2}} & \frac{1}{1-t_{1} t_{2}} & \frac{t_{1}}{\left(1-t_{1} t_{2}\right)^{2}} \\
\frac{t_{1}}{\left(1-t_{1}\right)^{2}} & \frac{1+t_{1}{ }^{2}}{\left(1-t_{1}\right)^{3}} & \frac{t_{2}}{\left(1-t_{1} t_{2}\right)^{2}} & \frac{1+t_{1} t_{2}}{\left(1-t_{1} t_{2}\right)^{3}} \\
\frac{1}{1-t_{1} t_{2}} & \frac{1+t_{1} t_{2}}{\left(1-t_{1} t_{2}\right)^{3}} & \frac{1}{1-t_{2}{ }^{2}} & \frac{t_{2}}{\left(1-t_{2}\right)^{2}} \\
\frac{t_{1}}{\left(1-t_{1} t_{2}\right)^{2}} & \frac{1+t_{1} t_{2}}{\left(1-t_{1} t_{2}\right)^{3}} & \frac{t_{2}}{\left(1-t_{2}\right)^{2}} & \frac{1+t_{2}{ }^{2}}{\left(1-t_{2}\right)^{3}}
\end{array}\right)
$$

We prove in the section 4 below that

$$
\operatorname{det} \Delta=\frac{\left(t_{1}-t_{2}\right)^{8}}{\left(1-t_{1}^{2}\right)^{4}\left(1-t_{2}^{2}\right)^{4}\left(1-t_{1} t_{2}\right)^{8}} \text {. }
$$

Let $\Omega$ be the two-by-two matrix obtained by removing the first and the third rows and columns from $\Delta^{-1}$. Then

$$
\Omega=\left(\begin{array}{ll}
A & B \\
B & C
\end{array}\right)
$$

where

$$
\begin{aligned}
& A=\left(1-t_{1} t_{2}\right)^{4}\left(1-t_{1}^{2}\right)^{3} /\left(t_{1}-t_{2}\right)^{4} \\
& B=\left(1-t_{1} t_{2}\right)^{3}\left(1-t_{1}^{2}\right)^{2}\left(1-t_{2}{ }^{2}\right)^{2} /\left(t_{1}-t_{2}\right)^{4} \\
& C=\left(1-t_{1} t_{2}\right)^{4}\left(1-t_{2}^{2}\right)^{3} /\left(t_{1}-t_{2}\right)^{4}
\end{aligned}
$$

By (2.6), the correlation function $K_{2}\left(t_{1}, t_{2}\right)$ is equal to

$$
K_{2}\left(t_{1}, t_{2}\right)=\frac{1}{4 \pi^{2} \sqrt{\operatorname{det} \Delta}} \int_{-\infty}^{\infty} \int_{-\infty}^{\infty}\left|y_{1} y_{2}\right| e^{-\frac{1}{2}(Y \Omega, Y)} d y_{1} d y_{2}
$$

where $Y=\left(y_{1}, y_{2}\right)$. Since

$$
\begin{aligned}
\int_{-\infty}^{\infty} \int_{-\infty}^{\infty}\left|y_{1} y_{2}\right| e^{-\frac{1}{2}\left(A y_{1}^{2}+2 B y_{1} y_{2}+C y_{2}^{2}\right)} d y_{1} d y_{2} \\
=\frac{4}{A C\left(1-\delta^{2}\right)}\left(1+\frac{\delta}{\sqrt{1-\delta^{2}}} \arcsin \delta\right), \quad \delta=\frac{B}{\sqrt{A C}}
\end{aligned}
$$

(see Appendix A), we obtain that

$$
\begin{aligned}
K_{2}\left(t_{1}, t_{2}\right)= & \frac{\left(t_{1}-t_{2}\right)^{2}}{\pi^{2}\left(1-t_{1} t_{2}\right)^{2}\left(1-t_{1}^{2}\right)\left(1-t_{2}^{2}\right)} \\
& +\frac{\left|t_{1}-t_{2}\right|}{\pi^{2}\left(1-t_{1} t_{2}\right)^{2} \sqrt{\left(1-t_{1}^{2}\right)\left(1-t_{2}^{2}\right)}} \arcsin \frac{\sqrt{\left(1-t_{1}^{2}\right)\left(1-t_{2}^{2}\right)}}{1-t_{1} t_{2}}
\end{aligned}
$$

Consider the correlation function $k_{2}\left(s_{1}, s_{2}\right)$ of the straightened zeroes $\zeta_{j}=\pi^{-1} \operatorname{artanh} \tau_{j}$. By (2.14),

$$
k_{2}\left(s_{1}, s_{2}\right)=\frac{K_{2}\left(t_{1}, t_{2}\right)}{p\left(t_{1}\right) p\left(t_{2}\right)}, \quad t_{1}=\tanh \left(\pi s_{1}\right), t_{2}=\tanh \left(\pi s_{2}\right) .
$$


Since

$$
p(t)=\frac{1}{\pi\left(1-t^{2}\right)},
$$

(see (1.5)), we obtain that

$$
\begin{aligned}
k_{2}\left(s_{1}, s_{2}\right) & =\frac{\left(t_{1}-t_{2}\right)^{2}}{\left(1-t_{1} t_{2}\right)^{2}}+\frac{\left|t_{1}-t_{2}\right| \sqrt{\left(1-t_{1}^{2}\right)\left(1-t_{2}^{2}\right)}}{\left(1-t_{1} t_{2}\right)^{2}} \arcsin \frac{\sqrt{\left(1-t_{1}^{2}\right)\left(1-t_{2}^{2}\right)}}{1-t_{1} t_{2}} \\
& =\tanh ^{2} \pi\left(s_{1}-s_{2}\right)+\frac{\left|\sinh \pi\left(s_{1}-s_{2}\right)\right|}{\cosh ^{2} \pi\left(s_{1}-s_{2}\right)} \arcsin \frac{1}{\cosh \pi\left(s_{1}-s_{2}\right)}
\end{aligned}
$$

Theorem 1.1 is proved.

\section{Higher order correlation functions for the Kac polynomial}

Let $f_{n}(t)$ be the Kac polynomial, and let $t_{1}, t_{2}, \ldots, t_{m}$ be $m \geq 3$ distinct points in the interval $(-1,1)$. Denote by $\Delta_{m}^{(n)}$ the covariance matrix of the Gaussian vector

$$
\left(f_{n}\left(t_{1}\right), f_{n}^{\prime}\left(t_{1}\right), \ldots, f_{n}\left(t_{m}\right), f_{n}^{\prime}\left(t_{m}\right)\right),
$$

and by $\Delta_{m}$ the limit of $\Delta_{m}^{(n)}$ as $n \rightarrow \infty$,

$$
\Delta_{m}=\lim _{n \rightarrow \infty} \Delta_{m}^{(n)}
$$

Then

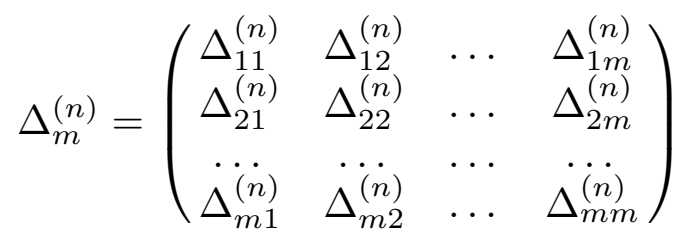

where

$$
\Delta_{i j}^{(n)}=\left(\begin{array}{ll}
\mathrm{E} f_{n}\left(t_{i}\right) f_{n}\left(t_{j}\right) & \mathrm{E} f_{n}\left(t_{i}\right) f_{n}^{\prime}\left(t_{j}\right) \\
\mathrm{E} f_{n}^{\prime}\left(t_{i}\right) f_{n}\left(t_{j}\right) & \mathrm{E} f_{n}^{\prime}\left(t_{i}\right) f_{n}^{\prime}\left(t_{j}\right)
\end{array}\right)
$$

and by (3.2),

$$
\Delta_{m}=\left(\begin{array}{cccc}
\Delta_{11} & \Delta_{12} & \ldots & \Delta_{1 m} \\
\Delta_{21} & \Delta_{22} & \ldots & \Delta_{2 m} \\
\ldots & \ldots & \ldots & \ldots \\
\Delta_{m 1} & \Delta_{m 2} & \ldots & \Delta_{m m}
\end{array}\right)
$$

where

$$
\Delta_{i j}=\left(\begin{array}{cc}
\frac{1}{1-t_{i} t_{j}} & \frac{t_{i}}{\left(1-t_{i} t_{j}\right)^{2}} \\
\frac{t_{j}}{\left(1-t_{i} t_{j}\right)^{2}} & \frac{1+t_{i} t_{j}}{\left(1-t_{i} t_{j}\right)^{3}}
\end{array}\right)
$$

[cf. (3.4)]. 
If $\Omega_{m}$ denotes the $m \times m$ matrix obtained by removing all the odd number rows and columns from $\Delta_{m}^{-1}$, then by $(2.6)$, the correlation function $K_{m}\left(t_{1}, \ldots, t_{m}\right)$ is equal to

$$
K_{m}\left(t_{1}, \ldots, t_{m}\right)=\frac{1}{(2 \pi)^{m} \sqrt{\operatorname{det} \Delta_{m}}} \int_{-\infty}^{\infty} \ldots \int_{-\infty}^{\infty}\left|y_{1} \ldots y_{m}\right| e^{-\frac{1}{2}\left(Y \Omega_{m}, Y\right)} d y_{1} \ldots d y_{m}
$$

where $Y=\left(y_{1}, \ldots, y_{m}\right)$. We have the following extension of the formula (3.6).

\section{Proposition 4.1}

$$
\operatorname{det} \Delta_{m}=\frac{\prod_{1 \leq i<j \leq m}\left(t_{i}-t_{j}\right)^{8}}{\prod_{i=1}^{m}\left(1-t_{i}^{2}\right)^{4} \prod_{1 \leq i<j \leq m}\left(1-t_{i} t_{j}\right)^{8}}
$$

The proof of Proposition 4.1 uses the following lemma.

Lemma 4.2 Let $f_{n}(t)(n \geq 3)$ be any random polynomial and $t_{1}, \ldots, t_{m}$ be any $m$ real numbers. Let $\Delta_{m}^{(n)}$ be the covariance matrix of the Gaussian random vector

$$
\left(f_{n}\left(t_{1}\right), f_{n}^{\prime}\left(t_{1}\right), \ldots, f_{n}\left(t_{m}\right), f_{n}^{\prime}\left(t_{m}\right)\right)
$$

which is defined in (4.2) and (4.3). Then

$$
\operatorname{det} \Delta_{m}^{(n)}=P_{n}\left(t_{1}, \ldots, t_{m}\right) \prod_{1 \leq i<j \leq m}\left(t_{i}-t_{j}\right)^{8}
$$

where $P_{n}\left(t_{1}, \ldots, t_{m}\right)$ is a polynomial.

Proof. To simplify notation we drop the indices $m, n$ in the matrix $\Delta_{m}^{(n)}$. We have

$$
\Delta=\left(\Delta_{i j}\right)_{i, j=1, \ldots, m}
$$

where

$$
\Delta_{i j}=\left(\begin{array}{ll}
\mathrm{E} f_{n}\left(t_{i}\right) f_{n}\left(t_{j}\right) & \mathrm{E} f_{n}\left(t_{i}\right) f_{n}^{\prime}\left(t_{j}\right) \\
\mathrm{E} f_{n}^{\prime}\left(t_{i}\right) f_{n}\left(t_{j}\right) & \mathrm{E} f_{n}^{\prime}\left(t_{i}\right) f_{n}^{\prime}\left(t_{j}\right)
\end{array}\right)
$$

In the following discussion we consider linear transformations of the matrix $\Delta$ which do not change its determinant. By substracting the first and second column of $\Delta_{i 1}$ from the first and second column of $\Delta_{i j}$, respectively, we get the matrix $\Delta^{(1)}$ with the $2 \times 2$ blocks

$$
\Delta_{i j}^{(1)}=\left(\begin{array}{ll}
\mathrm{E} f_{n}\left(t_{i}\right)\left(f_{n}\left(t_{j}\right)-f_{n}\left(t_{1}\right)\right) & \mathrm{E} f_{n}\left(t_{i}\right)\left(f_{n}^{\prime}\left(t_{j}\right)-f_{n}^{\prime}\left(t_{1}\right)\right) \\
\mathrm{E} f_{n}^{\prime}\left(t_{i}\right)\left(f_{n}\left(t_{j}\right)-f_{n}\left(t_{1}\right)\right) & \mathrm{E} f_{n}^{\prime}\left(t_{i}\right)\left(f_{n}^{\prime}\left(t_{j}\right)-f_{n}^{\prime}\left(t_{1}\right)\right)
\end{array}\right)
$$

Since $f_{n}(t)$ is a polynomial, we can take the factor $\left(t_{j}-t_{1}\right)$ out of the first two columns of the matrix $\Delta^{(1)}$, and this proves that $\operatorname{det} \Delta$ is divisible by $\left(t_{j}-t_{1}\right)^{2}$. Repeating the same operation on rows we get the factor $\left(t_{j}-t_{1}\right)^{4}$. How to get $\left(t_{j}-t_{1}\right)^{8}$ ? To that end let us 
subtract the second column of the matrix $\Delta_{i 1}^{(1)}=\Delta_{i 1}$ multiplied by $\left(t_{j}-t_{1}\right)$ from the first column of the matrix $\Delta_{i j}^{(1)}$. This produces the matrix

$$
\Delta_{i j}^{(2)}=\left(\begin{array}{ll}
\mathrm{E} f_{n}\left(t_{i}\right)\left[f_{n}\left(t_{j}\right)-f_{n}\left(t_{1}\right)-\left(t_{j}-t_{1}\right) f_{n}^{\prime}\left(t_{1}\right)\right] & \mathrm{E} f_{n}\left(t_{i}\right)\left[f_{n}^{\prime}\left(t_{j}\right)-f_{n}^{\prime}\left(t_{1}\right)\right] \\
\mathrm{E} f_{n}^{\prime}\left(t_{i}\right)\left[f_{n}\left(t_{j}\right)-f_{n}\left(t_{1}\right)-\left(t_{j}-t_{1}\right) f_{n}^{\prime}\left(t_{1}\right)\right] & \mathrm{E} f_{n}^{\prime}\left(t_{i}\right)\left[f_{n}^{\prime}\left(t_{j}\right)-f_{n}^{\prime}\left(t_{1}\right)\right]
\end{array}\right)
$$

Now we can take $\left(t_{j}-t_{1}\right)^{2}$ out of the first column and $\left(t_{j}-t_{1}\right)$ out of the second column of the matrix $\Delta^{(2)}$. Repeating the same operations over the rows we get that $\operatorname{det} \Delta$ is divisible by $\left(t_{j}-t_{1}\right)^{6}$. Finally, let us observe that by the Taylor formula

$$
f_{n}\left(t_{j}\right)-f_{n}\left(t_{1}\right)-\left(t_{j}-t_{1}\right) f_{n}\left(t_{1}\right)=\frac{\left(t_{j}-t_{1}\right)^{2}}{2} f_{n}^{\prime \prime}\left(t_{1}\right)+O\left(\left|t_{j}-t_{1}\right|^{3}\right),
$$

and

$$
f_{n}^{\prime}\left(t_{j}\right)-f_{n}^{\prime}\left(t_{1}\right)=\left(t_{j}-t_{1}\right) f_{n}^{\prime \prime}\left(t_{1}\right)+O\left(\left|t_{j}-t_{1}\right|^{2}\right),
$$

hence if we subtract the second column of the matrix $\Delta_{i j}^{(2)}$ multiplied by $\left(t_{j}-t_{1}\right) / 2$ from its first column, the difference is of the order of $\left|t_{j}-t_{1}\right|^{3}$, and we can take the factor $\left(t_{j}-t_{1}\right)^{3}$ out of the first column and $\left(t_{j}-t_{1}\right)$ out of the second column. This gives the factor $\left(t_{j}-t_{1}\right)^{4}$. The same factor is taken out of the raws, hence $\operatorname{det} \Delta$ is divisible by $\left(t_{j}-t_{1}\right)^{8}$. Similarly, it is divisible by $\left(t_{j}-t_{i}\right)^{8}$ for all $i \neq j$, and hence it is divisible by their product. Lemma 4.2 is proved.

Proof of Proposition 4.1. By (4.1), we have

$$
\operatorname{det} \Delta_{m}=\lim _{n \rightarrow \infty} \operatorname{det} \Delta_{m}^{(n)}
$$

for all $t_{1}, \ldots, t_{m}$ in the interval $(-1,1)$. In fact, the limit (4.13) holds for all complex $t_{1}, \ldots, t_{m}$ in the unit disk $\{|t|<1\}$, and the convergence is uniform on every disk $\{|t|<r\}$ where $r<1$. Hence by Lemma 4.2,

$$
\operatorname{det} \Delta_{m}=H\left(t_{1}, \ldots, t_{m}\right) \prod_{1 \leq i<j \leq m}\left(t_{i}-t_{j}\right)^{8}
$$

where $H\left(t_{1}, \ldots, t_{m}\right)$ is holomorphic in the unit disk.

Now, let us consider the expression of $\operatorname{det} \Delta_{m}$ in terms of the matrix elements $\delta_{i j}$ of $\Delta_{m}$, that is

$$
\operatorname{det} \Delta_{m}=\sum_{\sigma} \epsilon_{\sigma} \delta_{1 \sigma(1)} \ldots \delta_{n \sigma(2 m)}
$$

where $\sigma$ is a permutation of $\{1, \ldots, 2 m\}$ and $\epsilon_{m}= \pm 1$ depending on whether $\sigma$ is even or odd. The common denominator of the sum in (4.15) is

$$
\prod_{i=1}^{m}\left(1-t_{i}^{2}\right)^{4} \prod_{1 \leq i<j \leq m}\left(1-t_{i} t_{j}\right)^{8}
$$


Therefore by (4.14),

$$
\operatorname{det} \Delta_{m}=\frac{\prod_{1 \leq i<j \leq m}\left(t_{i}-t_{j}\right)^{8} C\left(t_{1}, \ldots, t_{m}\right)}{\prod_{i=1}^{m}\left(1-t_{i}^{2}\right)^{4} \prod_{1 \leq i<j \leq m}\left(1-t_{i} t_{j}\right)^{8}}
$$

where $C\left(t_{1}, \ldots, t_{m}\right)$ is a polynomial of $t_{1}, \ldots, t_{m}$. Observe that (4.17) holds for all points $t_{1}, \ldots, t_{m}$ in the unit disk, and so it can be extended to the whole complex plane. We are going to show that $C\left(t_{1}, \ldots, t_{m}\right)$ is a constant, and moreover, that

$$
C\left(t_{1}, \ldots, t_{m}\right)=1
$$

Let us look at the asymptotic behavior of det $\Delta_{m}$ as $t_{1} \rightarrow \infty$ while $t_{2}, \ldots, t_{m}$ are fixed. To see it more clearly, let us change $\Delta_{m}$ to $\Delta_{m}^{(1)}$ by substracting the $(2 i-1)$ th column and row from $(2 i)$ th column and row, respectively, for $i=1, \ldots, m$. Then

$$
\Delta_{m}^{(1)}=\left(\begin{array}{cccc}
\Delta_{11}^{(1)} & \Delta_{12}^{(1)} & \ldots & \Delta_{1 m}^{(1)} \\
\Delta_{21}^{(1)} & \Delta_{22}^{(1)} & \ldots & \Delta_{2 m}^{(1)} \\
\Delta_{m 1}^{(i)} & \ddot{\Delta}_{m 2}^{(i)} & \ldots & \ddot{⿴ 囗 十}_{m m}^{(1)}
\end{array}\right)
$$

where

$$
\Delta_{k k}^{(1)}=\left(\begin{array}{cc}
\frac{1}{1-t_{k}^{2}} & 0 \\
0 & \frac{1}{\left(1-t_{k}^{2}\right)^{3}}
\end{array}\right)
$$

for $k=1, \ldots, m$, and

$$
\Delta_{i j}^{(1)}=\left(\begin{array}{cc}
\frac{1}{1-t_{i} t_{j}} & \frac{t_{i}-t_{j}}{\left(1-t_{i} t_{j}\right)^{2}\left(1-t_{j}^{2}\right)} \\
\frac{t_{i}-t_{j}}{\left(1-t_{i} t_{j}\right)^{2}\left(1-t_{j}^{2}\right)} & \frac{2 t_{i} t_{j}+t_{i}^{2} t_{j}^{2}-2 t_{j}^{2}-2 t_{i}^{2}+1}{\left(1-t_{j}^{2}\right)\left(1-t_{i}^{2}\right)\left(1-t_{i} t_{j}\right)^{3}}
\end{array}\right)
$$

for $i \neq j$. The leading powers of the elements of $\Delta_{m}^{(1)}$, as $t_{1} \rightarrow \infty$, are

$$
\Delta_{m}^{(2)}=\left(\begin{array}{ccccccc}
1 / t_{1}^{2} & 0 & 1 / t_{1} & 1 / t_{1} & \ldots & 1 / t_{1} & 1 / t_{1} \\
0 & 1 / t_{1}^{6} & 1 / t_{1}^{3} & 1 / t_{1}^{3} & \ldots & 1 / t_{1}^{3} & 1 / t_{1}^{3} \\
1 / t_{1} & 1 / t_{1}^{3} & * & * & \ldots & * & * \\
1 / t_{1} & 1 / t_{1}^{3} & * & * & \ldots & * & * \\
\ldots & \ldots & \ldots & \ldots & \ldots & \ldots & \ldots \\
1 / t_{1} & 1 / t_{1}^{3} & * & * & \ldots & * & * \\
1 / t_{1} & 1 / t_{1}^{3} & * & * & \ldots & * & *
\end{array}\right)
$$

where $^{*}$ 's stand for the terms of the order of $O(1)$. Therefore

$$
\operatorname{det} \Delta_{m}=O\left(\frac{1}{t_{1}^{8}}\right), \quad t_{1} \rightarrow \infty
$$


By (4.17),

$$
\operatorname{det} \Delta_{m} \sim \frac{\text { const. } C\left(t_{1}, \ldots, t_{m}\right)}{t_{1}^{8}}, \quad t_{1} \rightarrow \infty,
$$

hence $C\left(t_{1}, \ldots, t_{m}\right)$ is constant in $t_{1}$. The same argument on $t_{2}, \ldots, t_{m}$ shows that it is independent of $t_{2}, \ldots, t_{m}$, so it is indeed a constant, say $C_{m}$, i.e.,

$$
\operatorname{det} \Delta_{m}=\frac{C_{m} \prod_{1 \leq i<j \leq m}\left(t_{i}-t_{j}\right)^{8}}{\prod_{i=1}^{m}\left(1-t_{i}^{2}\right)^{4} \prod_{1 \leq i<j \leq m}\left(1-t_{i} t_{j}\right)^{8}}
$$

To prove that $C_{m}=1$, let us consider the asymptotic behavior of det $\Delta_{m}$ as $t_{1} \rightarrow 1$ with $t_{2}, \ldots, t_{m}$ fixed and close to zero. Then on the one hand, we have from (4.28) that

$$
\lim _{t_{1} \rightarrow 1}\left(1-t_{1}^{2}\right)^{4} \operatorname{det} \Delta_{m}=\frac{C_{m}}{C_{m-1}} \operatorname{det} \Delta_{m-1},
$$

On the other hand,

$$
\Delta_{m}^{(1)}=\left(\begin{array}{ccccc}
\frac{1}{\left(1-t_{1}^{2}\right)} & 0 & * & \ldots & 0 \\
0 & \frac{1}{\left(1-t_{1}^{2}\right)^{3}} & * & \ldots & * \\
* & * & & & \\
\ldots & \ldots & & \Delta_{m-1} & \\
* & * & & &
\end{array}\right)
$$

where the terms $*$ are regular at $t_{1}=1$. Hence the leading term of the Laurent series of $\operatorname{det} \Delta_{m}$ at $t_{1}=1$ is $\left(1-t_{1}\right)^{-4} \operatorname{det} \Delta_{m-1}$, which shows that

$$
\frac{C_{m}}{C_{m-1}} \operatorname{det} \Delta_{m-1}=\operatorname{det} \Delta_{m-1}
$$

Thus $C_{m}=C_{m-1}$. Repeating this argument we get that

$$
C_{m}=C_{m-1}=\cdots=C_{2}=C_{1}=1
$$

Therefore $C_{m}=1$. Proposition 4.1 is proved.

Similarly we prove the following proposition.

\section{Proposion 4.3.}

$$
\Omega_{m}=\left(\begin{array}{cccc}
\omega_{11} & \omega_{12} & \ldots & \omega_{1 m} \\
\omega_{21} & \omega_{22} & \ldots & \omega_{2 m} \\
\ldots & \ldots & \ldots & \ldots \\
\omega_{m 1} & \omega_{m 2} & \ldots & \omega_{m m}
\end{array}\right)
$$

with

$$
\omega_{i i}=\frac{\left(1-t_{i}^{2}\right)^{3} \prod_{j \neq i}\left(1-t_{i} t_{j}\right)^{4}}{\prod_{j \neq i}\left(t_{i}-t_{j}\right)^{4}}
$$


and

$$
\omega_{i j}=\frac{\left(1-t_{i}^{2}\right)^{2}\left(1-t_{j}^{2}\right)^{2} \prod_{k \neq i}\left(1-t_{i} t_{k}\right)^{2} \prod_{k \neq j}\left(1-t_{j} t_{k}\right)^{2}}{\left(1-t_{i} t_{j}\right) \prod_{k \neq i}\left(t_{i}-t_{k}\right)^{2} \prod_{k \neq j}\left(t_{j}-t_{k}\right)^{2}}, \quad i \neq j .
$$

Put now

$$
t_{i}=\tanh \left(\pi s_{i}\right) \quad i=1, \ldots, m .
$$

Then by (4.29) and (4.30),

$$
\omega_{i j}=\left(1-t_{i}^{2}\right)^{3 / 2}\left(1-t_{j}^{2}\right)^{3 / 2} \frac{\prod_{k \neq i} \operatorname{coth}^{2} \pi\left(s_{i}-s_{k}\right) \prod_{k \neq j} \operatorname{coth}^{2} \pi\left(s_{j}-s_{k}\right)}{\cosh \pi\left(s_{i}-s_{j}\right)} .
$$

In addition, by (4.7),

$$
\operatorname{det} \Delta_{m}=\frac{\prod_{1 \leq i<j \leq m} \tanh ^{8} \pi\left(s_{i}-s_{j}\right)}{\prod_{i=1}^{m}\left(1-t_{i}^{2}\right)^{4}}
$$

By (2.14),

$$
k_{m}\left(s_{1}, \ldots, s_{m}\right)=\frac{K_{m}\left(t_{1}, \ldots, t_{m}\right)}{\prod_{i=1}^{m} p\left(t_{i}\right)}, \quad p(t)=\frac{1}{\pi\left(1-t^{2}\right)} .
$$

Now let us substitute $y_{i}$ 's in (4.6) by

$$
\frac{y_{i}}{\left(1-t_{i}^{2}\right)^{3 / 2}}, \quad i=1, \ldots, m
$$

then

$$
\begin{aligned}
K_{m}\left(t_{1}, \ldots, t_{m}\right) & =\frac{\prod_{i=1}^{m} p\left(t_{i}\right)}{2^{m} \prod_{1 \leq i<j \leq m} \tanh ^{4} \pi\left(s_{i}-s_{j}\right)} \\
& \times \int_{-\infty}^{\infty} \ldots \int_{-\infty}^{\infty}\left|y_{1} \ldots y_{m}\right| e^{-\frac{1}{2}\left(Y \Sigma_{m}, Y\right)} d y_{1} \ldots d y_{m}
\end{aligned}
$$

where

$$
\Sigma_{m}=\left(\begin{array}{cccc}
\sigma_{11} & \sigma_{12} & \ldots & \sigma_{1 m} \\
\sigma_{21} & \sigma_{22} & \ldots & \sigma_{2 m} \\
\ldots & \ldots & \ldots & \ldots \\
\sigma_{m 1} & \sigma_{m 2} & \ldots & \sigma_{m m}
\end{array}\right)
$$

with

$$
\sigma_{i j}=\frac{\prod_{k \neq i} \operatorname{coth}^{2} \pi\left(s_{i}-s_{k}\right) \prod_{k \neq j} \operatorname{coth}^{2} \pi\left(s_{j}-s_{k}\right)}{\cosh \pi\left(s_{i}-s_{j}\right)}
$$

Now substitute $y_{i}$ 's in the formula (4.36) by

$$
\frac{y_{i}}{\prod_{j \neq i} \operatorname{coth}^{2} \pi\left(s_{i}-s_{j}\right)}, \quad i=1, \ldots, m
$$


then

$$
K_{m}\left(t_{1}, \ldots, t_{m}\right)=2^{-m} \prod_{i=1}^{m} p\left(t_{i}\right) \int_{-\infty}^{\infty} \ldots \int_{-\infty}^{\infty}\left|y_{1} \ldots y_{m}\right| e^{-\frac{1}{2}\left(Y \Gamma_{m}, Y\right)} d y_{1} \ldots d y_{m}
$$

where

$$
\Gamma_{m}=\left(\frac{1}{\cosh \pi\left(s_{i}-s_{j}\right)}\right)_{i, j=1}^{m}
$$

Thus

$$
k_{m}\left(s_{1}, \ldots, s_{m}\right)=2^{-m} \int_{-\infty}^{\infty} \ldots \int_{-\infty}^{\infty}\left|y_{1} \ldots y_{m}\right| e^{-\frac{1}{2}\left(Y \Gamma_{m}, Y\right)} d y_{1} \ldots d y_{m}
$$

Theorem 1.2 is proved.

Proof of Theorem 1.3. Let

$$
f_{n}(t)=c_{0}+c_{1} t+\cdots+c_{n-1} t^{n-1}+c_{n} t^{n}, \quad g_{n}(t)=c_{0} t^{n}+c_{1} t^{n-1}+\cdots+c_{n-1} t+c_{n} .
$$

Then if $\tau_{k} \neq 0$ is a zero of $f_{n}(t)$ then $\tau_{k}^{-1}$ is a zero of $g_{n}(t)$. Hence if $1<a<b$ then

$$
\xi_{f}(a, b)=\xi_{g}\left(a^{-1}, b^{-1}\right)
$$

where $\xi_{f}(a, b)$ is the number of zeros of $f_{n}(t)$ in the interval $(a, b)$. Observe that the distribution of the vector of coefficients $\left(c_{0}, \ldots, c_{n}\right)$ coincides with the one of the vector $\left(c_{n}, \ldots, c_{0}\right)$. Hence

$$
\begin{aligned}
\mathrm{E}\left[\xi_{f}\left(a_{1}, b_{1}\right) \ldots \xi_{f}\left(a_{m}, b_{m}\right)\right] & =\mathrm{E}\left[\xi_{g}\left(a_{1}^{-1}, b_{1}^{-1}\right) \ldots \xi_{g}\left(a_{m}^{-1}, b_{m}^{-1}\right)\right] \\
& =\mathrm{E}\left[\xi_{f}\left(a_{1}^{-1}, b_{1}^{-1}\right) \ldots \xi_{f}\left(a_{m}^{-1}, b_{m}^{-1}\right)\right]
\end{aligned}
$$

Take $a_{j}=t_{j}$ and $b_{j}=t_{j}+\Delta t_{j}, j=1, \ldots, m$, and get $\Delta t_{j} \rightarrow 0$. Since

$$
\left|a^{-1}-b^{-1}\right|=a^{-2}|a-b|+O\left(|a-b|^{2}\right), \quad a \rightarrow b,
$$

we deduce that

$$
\frac{K_{n m}\left(t_{1}^{-1}, \ldots, t_{m}^{-1}\right)}{t_{1}^{2} \ldots t_{m}^{2}}=K_{n m}\left(t_{1}, \ldots, t_{m}\right)
$$

Hence

$$
\frac{K_{n m}\left(t_{1}^{-1}, \ldots, t_{m}^{-1}\right)}{p\left(t_{1}^{-1}\right) \ldots p\left(t_{m}^{-1}\right)}=\frac{K_{n m}\left(t_{1}, \ldots, t_{m}\right)}{p\left(t_{1}\right) \ldots p\left(t_{m}\right)}
$$

because

$$
p\left(t^{-1}\right)=\frac{1}{\pi\left|1-t^{-2}\right|}=t^{2} p(t)
$$


This proves that

$$
k_{n m}^{\text {out }}\left(s_{1}, \ldots, s_{m}\right)=k_{n m}\left(s_{1}, \ldots, s_{m}\right) .
$$

Theorem 1.3 is proved.

Proof of Theorem 1.4. Let $\left|t_{1}\right|, \ldots,\left|t_{l}\right|<1$ and $\left|t_{l+1}\right|, \ldots,\left|t_{m}\right|>1$. Denote by $D_{n m}\left(x_{1}, y_{1}, \ldots, x_{m}, y_{m} ; t_{1}, \ldots t_{m}\right)$ the joint distribution density of the vector

$$
\left(f_{n}\left(t_{1}\right), f_{n}\left(t_{1}\right), \ldots, f_{n}\left(t_{l}\right), f_{n}^{\prime}\left(t_{l}\right), g_{n}\left(t_{l+1}\right), g_{n}^{\prime}\left(t_{l+1}\right), \ldots g_{n}\left(t_{m}\right), g_{n}^{\prime}\left(t_{m}\right)\right)
$$

Then

$$
\begin{aligned}
K_{n m}\left(t_{1}, \ldots, t_{m}\right) & =\int_{-\infty}^{\infty} \cdots \int_{-\infty}^{\infty}\left|y_{1} \ldots y_{m}\right| \\
& \times D_{n m}\left(0, y_{1}, \ldots, 0, y_{m} ; t_{1}, \ldots, t_{l}, t_{l+1}^{-1}, \ldots, t_{m}^{-1}\right) d y_{1} \ldots d y_{m}
\end{aligned}
$$

The covariance

$$
\mathrm{E} f_{n}\left(t_{i}\right) g_{n}\left(t_{j}^{-1}\right)=t_{i}^{n}+t_{i}^{n-1} t_{j}^{-1}+\cdots+t_{i} t_{j}^{1-n}+t_{j}^{-n}, \quad 1 \leq i \leq l<j \leq m
$$

goes to 0 as $n \rightarrow \infty$, together with the partial derivatives in $t_{i}, t_{j}$, while

$$
\begin{aligned}
& \lim _{n \rightarrow \infty} \operatorname{E} f_{n}\left(t_{i}\right) f_{n}\left(t_{j}\right)=\frac{1}{1-t_{i} t_{j}}, \quad 1 \leq i, j \leq l, \\
& \lim _{n \rightarrow \infty} \operatorname{E} g_{n}\left(t_{i}^{-1}\right) g_{n}\left(t_{j}^{-1}\right)=\frac{1}{1-t_{i}^{-1} t_{j}^{-1}}, \quad l+1 \leq i, j \leq m .
\end{aligned}
$$

This proves that the limiting Gaussian kernel $D_{m}=\lim _{n \rightarrow \infty} D_{n m}$ is factored,

$$
\begin{array}{r}
D_{m}\left(x_{1}, y_{1}, \ldots x_{m}, y_{m} ; t_{1}, \ldots, t_{l}, t_{l+1}^{-1}, \ldots, t_{m}^{-1}\right)=D_{l}(f)\left(x_{1}, y_{1}, \ldots, x_{l}, y_{l} ; t_{1}, \ldots, t_{l}\right) \\
\times D_{m-l}(g)\left(x_{l+1}, y_{l+1}, \ldots, x_{m}, y_{m} ; t_{l+1}^{-1}, \ldots, t_{m}^{-1}\right) .
\end{array}
$$

Hence the correlation function $K_{m}$ is also factored. Theorem 1.4 is proved. 


\section{Correlations between Zeros of the SO(2) Random Polynomial}

In the following two sections we discuss the correlation functions of real zeros and the variance of the number of real zeros of the $\mathrm{SO}(2)$ random polynomial. Let $f_{n}(t)$ be a $\mathrm{SO}(2)$ random polynomial, that is

$$
f_{n}(t)=\sum_{k=0}^{n} c_{k} t^{k}
$$

where $\left\{c_{k}\right\}$ are real independent Gaussian random variables with

$$
\mathrm{E} c_{k}=0, \quad \mathrm{E} c_{k}^{2}=\sigma_{k}^{2}=\left(\begin{array}{l}
n \\
k
\end{array}\right) .
$$

In this case (1.4) reduces to

$$
\begin{aligned}
& A_{n}(t)=\left(1+t^{2}\right)^{n}, \\
& B_{n}(t)=n t\left(1+t^{2}\right)^{n-1}, \\
& C_{n}(t)=n\left(1+n t^{2}\right)\left(1+t^{2}\right)^{n-2},
\end{aligned}
$$

which gives

$$
p_{n}(t)=\frac{\sqrt{n}}{\pi\left(1+t^{2}\right)}
$$

(see $[\mathrm{BS}],[\mathrm{EK}],[\mathrm{BBL} 1]$, and others). The average value of real zeros is

$$
\mathrm{E} \#\left\{k: \tau_{k} \in \mathbb{R}\right\}=\int_{-\infty}^{\infty} p_{n}(t) d t=\sqrt{n},
$$

and the normalized density,

$$
\frac{p_{n}(t)}{\sqrt{n}}=\frac{1}{\pi\left(1+t^{2}\right)},
$$

is the Cauchy distribution density. Observe that both (5.4) and (5.5) are exact relations for all $n$. Let us compute the two-point correlation function $K_{n 2}\left(t_{1}, t_{2}\right)$.

Define

$$
\varphi_{n}(t)=\frac{f_{n}(t)}{\left(1+t^{2}\right)^{n / 2}} .
$$

Then the real zeros of $\varphi_{n}(t)$ coincide with those of $f_{n}(t)$. Hence, similar to (2.6), we can write that

$$
K_{n 2}\left(t_{1}, t_{2}\right)=\int_{-\infty}^{\infty} \int_{-\infty}^{\infty}\left|y_{1} y_{2}\right| D_{n 2}\left(0, y_{1}, 0, y_{2} ; t_{1}, t_{2}\right) d y_{1} d y_{2},
$$


where $D_{n 2}\left(x_{1}, y_{1}, x_{2}, y_{2} ; t_{1}, t_{2}\right)$ is the distribution density function of the vector

$$
\left(\varphi_{n}\left(t_{1}\right), \varphi_{n}^{\prime}\left(t_{1}\right), \varphi_{n}\left(t_{2}\right), \varphi_{n}^{\prime}\left(t_{2}\right)\right)
$$

Observe that

$$
\mathrm{E} \varphi_{n}\left(t_{1}\right) \varphi_{n}\left(t_{2}\right)=\rho_{n}\left(t_{1}, t_{2}\right) \equiv \frac{\left(1+t_{1} t_{2}\right)^{n}}{\left(1+t_{1}^{2}\right)^{n / 2}\left(1+t_{2}^{2}\right)^{n / 2}}
$$

and

$$
\begin{aligned}
& \mathrm{E} \varphi_{n}^{\prime}\left(t_{1}\right) \varphi_{n}\left(t_{2}\right)=\frac{\partial \rho_{n}}{\partial t_{1}}\left(t_{1}, t_{2}\right)=n \rho_{n} \frac{\left(t_{2}-t_{1}\right)}{\left(1+t_{1} t_{2}\right)\left(1+t_{1}^{2}\right)} \\
& \mathrm{E} \varphi_{n}^{\prime}\left(t_{1}\right) \varphi_{n}^{\prime}\left(t_{2}\right)=\frac{\partial^{2} \rho_{n}}{\partial t_{1} \partial t_{2}}\left(t_{1}, t_{2}\right) \\
& =-n^{2} \rho_{n} \frac{\left(t_{2}-t_{1}\right)^{2}}{\left(1+t_{1} t_{2}\right)^{2}\left(1+t_{1}^{2}\right)\left(1+t_{2}^{2}\right)}+n \rho_{n} \frac{1}{\left(1+t_{1} t_{2}\right)^{2}} .
\end{aligned}
$$

Define the random variable $\psi_{n}(t)$ as

$$
\psi_{n}(t)=\frac{\varphi_{n}^{\prime}(t)\left(1+t^{2}\right)}{\sqrt{n}} .
$$

Let $D_{n 2}\left(x_{1}, y_{1}, x_{2}, y_{2} ; t_{1}, t_{2}\right)$ be the distribution density function of the random vector $\left(\varphi_{n}\left(t_{1}\right), \psi_{n}\left(t_{1}\right), \varphi_{n}\left(t_{2}\right), \psi_{n}\left(t_{2}\right)\right)$. Then after a change of variables, (5.8) is rewritten as

$$
\frac{K_{n 2}\left(t_{1}, t_{2}\right)}{p_{n}\left(t_{1}\right) p_{n}\left(t_{2}\right)}=\pi^{2} \int_{-\infty}^{\infty} \int_{-\infty}^{\infty}\left|y_{1} y_{2}\right| D_{n 2}\left(0, y_{1}, 0, y_{2} ; t_{1}, t_{2}\right) d y_{1} d y_{2}
$$

From (5.10),

$$
\begin{aligned}
& \mathrm{E} \psi_{n}\left(t_{1}\right) \varphi_{n}\left(t_{2}\right)=\rho_{n} \frac{\sqrt{n}\left(t_{2}-t_{1}\right)}{\left(1+t_{1} t_{2}\right)} \\
& \mathrm{E} \psi_{n}\left(t_{1}\right) \psi_{n}\left(t_{2}\right)=\rho_{n}\left[-\frac{n\left(t_{2}-t_{1}\right)^{2}}{\left(1+t_{1} t_{2}\right)^{2}}+\frac{\left(1+t_{1}^{2}\right)\left(1+t_{2}^{2}\right)}{\left(1+t_{1} t_{2}\right)^{2}}\right]
\end{aligned}
$$

hence the covariance matrix of the vector $\left(\varphi_{n}\left(t_{1}\right), \psi_{n}\left(t_{1}\right), \varphi_{n}\left(t_{2}\right), \psi_{n}\left(t_{2}\right)\right)$ is

$$
\Delta_{n}=\left(\begin{array}{cccc}
1 & 0 & \rho_{n} & \rho_{n} \frac{\sqrt{n}\left(t_{1}-t_{2}\right)}{\left(1+t_{1} t_{2}\right)} \\
0 & 1 & \rho_{n} \frac{\sqrt{n}\left(t_{2}-t_{1}\right)}{\left(1+t_{1} t_{2}\right)} & \rho_{n} a \\
\rho_{n} & \rho_{n} \frac{\sqrt{n}\left(t_{2}-t_{1}\right)}{\left(1+t_{1} t_{2}\right)} & 1 & 0 \\
\rho_{n} \frac{\sqrt{n}\left(t_{1}-t_{2}\right)}{\left(1+t_{1} t_{2}\right)} & \rho_{n} a & 0 & 1
\end{array}\right)
$$

where

$$
a=a\left(t_{1}, t_{2}\right)=-\frac{n\left(t_{2}-t_{1}\right)^{2}}{\left(1+t_{1} t_{2}\right)^{2}}+\frac{\left(1+t_{1}^{2}\right)\left(1+t_{2}^{2}\right)}{\left(1+t_{1} t_{2}\right)^{2}} .
$$


Suppose that $t_{1}$ and $t_{2}$ are two distinct fixed points. Then as $n \rightarrow \infty$, the quantity

$$
\left|\rho_{n}\right|=\left[1-\frac{\left(t_{1}-t_{2}\right)^{2}}{\left(1+t_{1}^{2}\right)\left(1+t_{2}^{2}\right)}\right]^{n / 2}
$$

goes to 0 exponentially fast, and hence $\Delta_{n}$ approaches the unit matrix exponentially fast. By (5.12) this implies that

$$
\lim _{n \rightarrow \infty} \frac{K_{n 2}\left(t_{1}, t_{2}\right)}{p_{n}\left(t_{1}\right) p_{n}\left(t_{2}\right)}=1
$$

and the rate of convergence is exponential. In the same way we obtain that if $t_{1}, \ldots, t_{m}$ are $m$ distinct fixed points then

$$
\frac{K_{n m}\left(t_{1}, \ldots, t_{m}\right)}{p_{n}\left(t_{1}\right) \ldots p_{n}\left(t_{m}\right)}=\pi^{m} \int_{-\infty}^{\infty} \ldots \int_{-\infty}^{\infty}\left|y_{1} \ldots y_{m}\right| D_{n m}\left(0, y_{1}, \ldots 0, y_{m} ; t_{1}, \ldots, t_{m}\right) d y_{1} \ldots d y_{m}
$$

where $D_{n m}\left(x_{1}, y_{1}, \ldots x_{m}, y_{m} ; t_{1}, \ldots, t_{m}\right)$ is a $(2 m) \times(2 m)$ Gaussian density with the covariance matrix

$$
\Delta_{n}=\left(\Delta_{i j}^{(n)}\right)_{i, j=1, \ldots, m}
$$

where

$$
\Delta_{i i}^{(n)}=\left(\begin{array}{ll}
1 & 0 \\
0 & 1
\end{array}\right), \quad \Delta_{i j}^{(n)}=\rho_{n}\left(t_{i}, t_{j}\right)\left(\begin{array}{cc}
1 & \frac{\sqrt{n}\left(t_{i}-t_{j}\right)}{\left(1+t_{i} t_{j}\right)} \\
\frac{\sqrt{n}\left(t_{j}-t_{i}\right)}{\left(1+t_{1} t_{2}\right)} & a\left(t_{i}, t_{j}\right)
\end{array}\right)
$$

where $\rho_{n}\left(t_{1}, t_{2}\right)$ and $a\left(t_{1}, t_{2}\right)$ are defined in (5.9) and (5.15), respectively. For fixed different $t_{1}, \ldots, t_{m}$ the matrix $\Delta_{n}$ approaches the unit matrix exponentially fast, and this implies that

$$
\lim _{n \rightarrow \infty} \frac{K_{n m}\left(t_{1}, \ldots, t_{m}\right)}{p_{n}\left(t_{1}\right) \ldots p_{n}\left(t_{m}\right)}=1
$$

and the rate of convergence is exponential. This means the independence of the distribution of real zeros at distinct fixed points.

The formula (5.17) is simplified if we make the change of variables $t=\tan \theta$ (stereographic projection). Consider therefore the random function

$$
g_{n}(\theta)=\sum_{j=0}^{n} c_{k} \sin ^{k}(\theta) \cos ^{n-k}(\theta), \quad-\frac{\pi}{2}<\theta \leq \frac{\pi}{2} .
$$

Then

$$
g_{n}(\theta)=\cos ^{n} \theta f_{n}(\tan \theta),
$$

hence if $\left\{\tau_{j}\right\}$ are zeros of $f_{n}(t)$ then

$$
\left\{\eta_{j}=\arctan \tau_{j}\right\}
$$


are zeros of $g_{n}(\theta)$. When $c_{n}=0, g_{n}(\theta)$ has an extra zero $\eta=\pi / 2$. Since the probability that $c_{n}=0$ is equal to zero, the joint probability distributions of zeros of the functions $g_{n}(\theta)$ and $f_{n}(\tan \theta)$ coincide. By $(5.6)$ the zeros $\left\{\eta_{j}\right\}$ are uniformly distributed on the interval $[-\pi / 2, \pi / 2]$. The function $g_{n}(\theta)$ is a Gaussian random function with zero mean and the covariance function

$$
\mathrm{E} g_{n}\left(\theta_{1}\right) g_{n}\left(\theta_{2}\right)=\sum_{k=0}^{n}\left(\begin{array}{l}
n \\
k
\end{array}\right) \sin ^{k} \theta_{1} \cos ^{n-k} \theta_{1} \sin ^{k} \theta_{2} \cos ^{n-k} \theta_{2}=\cos ^{n}\left(\theta_{1}-\theta_{2}\right)
$$

The function $g_{n}(\theta)$ is periodic of period $\pi$ if $n$ is even, and it is periodic of period $2 \pi$ if $n$ is odd. It is convenient to consider $g_{n}(\theta)$ on the circle $S^{1}=\mathbb{R} /(2 \pi) \mathbb{Z}$ of the length $2 \pi$. This circle is the covering space of the original circle $\mathbb{R} / \pi \mathbb{Z}$. If $g_{n}(\theta)=0$ then $g_{n}(\theta+\pi)=0$ as well. On $S^{1}$ the distribution of $g_{n}(\theta)$ is invariant with respect to the shift

$$
\theta \rightarrow \alpha+\theta \bmod 2 \pi
$$

hence it is $\mathrm{SO}(2)$-invariant.

Let $K_{n m}\left(\theta_{1}, \ldots, \theta_{m}\right)$ be the correlation function of the zeros $\left\{\eta_{j}\right\}$ of $g_{n}(\theta)$. Assume that

$$
\theta_{j}-\theta_{k} \neq 0 \quad \bmod \pi, \quad 1 \leq j, k \leq m
$$

Then (5.17) gives that

$$
\frac{K_{n m}\left(\theta_{1}, \ldots, \theta_{m}\right)}{\left(\pi^{-1} \sqrt{n}\right)^{m}}=\pi^{m} \int_{-\infty}^{\infty} \ldots \int_{-\infty}^{\infty}\left|y_{1} \ldots y_{m}\right| D_{n m}\left(0, y_{1}, \ldots 0, y_{m} ; \theta_{1}, \ldots, \theta_{m}\right) d y_{1} \ldots d y_{m}
$$

where $D_{n m}\left(x_{1}, y_{1}, \ldots x_{m}, y_{m} ; \theta_{1}, \ldots, \theta_{m}\right)$ is a $(2 m) \times(2 m)$ Gaussian density with the covariance matrix

$$
\Delta_{n}=\left(\Delta_{i j}^{(n)}\right)_{i, j=1, \ldots, m}
$$

where

$$
\Delta_{i j}^{(n)}=\cos ^{n}\left(\theta_{i}-\theta_{j}\right)\left(\begin{array}{cc}
1 & \sqrt{n} \tan \left(\theta_{i}-\theta_{j}\right) \\
\sqrt{n} \tan \left(\theta_{j}-\theta_{i}\right) & -n \tan ^{2}\left(\theta_{i}-\theta_{j}\right)+\cos ^{-2}\left(\theta_{i}-\theta_{j}\right)
\end{array}\right)
$$

Observe that $D_{n m}\left(x_{1}, y_{1}, \ldots x_{m}, y_{m} ; \theta_{1}, \ldots, \theta_{m}\right)$ is the probability distribution density of the vector

$$
\left(g_{n}\left(\theta_{1}\right), \frac{g_{n}^{\prime}\left(\theta_{1}\right)}{\sqrt{n}}, \ldots, g_{n}\left(\theta_{m}\right), \frac{g_{n}^{\prime}\left(\theta_{m}\right)}{\sqrt{n}}\right)
$$

and it is nondegenerate provided that $n \geq 2 m-1$ and (5.22) holds.

Consider now the scaling limit of the correlation functions. The straightened zeros are

$$
\zeta_{j}=\frac{\eta_{j} \sqrt{n}}{\pi}
$$


They are uniformly distributed on the circle of the length $2 \sqrt{n}$. The limit $m$-point correlation function of $\left\{\zeta_{j}\right\}$ is

$$
k_{m}\left(s_{1}, \ldots, s_{m}\right)=\lim _{n \rightarrow \infty} \frac{K_{n m}\left(\theta_{1}, \ldots, \theta_{m}\right)}{\left(\pi^{-1} \sqrt{n}\right)^{m}}, \quad \theta_{i}=\frac{s_{i} \pi}{\sqrt{n}}
$$

Let us find $k_{m}\left(s_{1}, \ldots, s_{m}\right)$. We have that

$$
\lim _{n \rightarrow \infty} \cos ^{n}\left(\frac{\left(s_{i}-s_{j}\right) \pi}{\sqrt{n}}\right)=e^{-\pi^{2}\left(s_{i}-s_{j}\right)^{2} / 2}
$$

and by (5.24),

$$
\lim _{n \rightarrow \infty} \Delta_{n}=\Delta=\left(\Delta_{i j}\right)_{i, j=1, \ldots, m}
$$

with

$$
\Delta_{i j}=e^{-\pi^{2}\left(s_{i}-s_{j}\right)^{2} / 2}\left(\begin{array}{cc}
1 & \pi\left(s_{i}-s_{j}\right) \\
\pi\left(s_{j}-s_{i}\right) & 1-\pi^{2}\left(s_{i}-s_{j}\right)^{2}
\end{array}\right)
$$

By (5.23) this gives that

$$
k_{m}\left(s_{1}, \ldots, s_{m}\right)=\pi^{m} \int_{-\infty}^{\infty} \ldots \int_{-\infty}^{\infty}\left|y_{1} \ldots y_{m}\right| d_{m}\left(0, y_{1}, \ldots 0, y_{m} ; s_{1}, \ldots, s_{m}\right) d y_{1} \ldots d y_{m}
$$

and $d_{m}\left(x_{1}, y_{1}, \ldots x_{m}, y_{m} ; s_{1}, \ldots, s_{m}\right)$ is a Gaussian density with the covariance matrix $\Delta$. Let $\Omega$ be $m \times m$ matrix obtained by deleting all odd rows and odd columns from the matrix $\Delta^{-1}$. Then we can write $(5.28)$ as

$$
k_{m}\left(s_{1}, \ldots, s_{m}\right)=\frac{1}{2^{m}(\operatorname{det} \Delta)^{1 / 2}} \int_{-\infty}^{\infty} \ldots \int_{-\infty}^{\infty}\left|y_{1} \ldots y_{m}\right| e^{-\frac{1}{2}(Y \Omega, Y)} d y_{1} \ldots d y_{m}
$$

In Appendix $\mathrm{C}$ below we prove that

$$
\Delta>0 \quad \text { if } \quad s_{i} \neq s_{j}, 1 \leq i<j \leq m
$$

so the formula (5.29) is well-defined when the points $s_{i}$ are distinct.

For $m=2,(5.29)$ reduces to

$$
k_{2}\left(s_{1}, s_{2}\right)=\frac{1}{4 \pi^{2}(\operatorname{det} \Delta)^{1 / 2}} \int_{-\infty}^{\infty} \int_{-\infty}^{\infty}\left|y_{1} y_{2}\right| e^{-\frac{1}{2}(Y \Omega, Y)} d y_{1} d y_{2} .
$$

where

$$
\Delta=\left(\begin{array}{cccc}
1 & 0 & e^{-s^{2} / 2} & s e^{-s^{2} / 2} \\
0 & 1 & -s e^{-s^{2} / 2} & \left(1-s^{2}\right) e^{-s^{2} / 2} \\
e^{-s^{2} / 2} & -s e^{-s^{2} / 2} & 1 & 0 \\
s e^{-s^{2} / 2} & \left(1-s^{2}\right) e^{-s^{2} / 2} & 0 & 1
\end{array}\right), \quad s=\pi\left(s_{1}-s_{2}\right)
$$


and

$$
\Omega=\left(\Delta^{-1}\right)_{\{2,4\}},
$$

i.e., $\Omega$ is the $2 \times 2$-submatrix of $\Delta^{-1}$ at the second and the fourth rows and columns. Observe that

$$
\operatorname{det} \Omega=\frac{\operatorname{det} \Delta_{\{1,3\}}}{\operatorname{det} \Delta}=\frac{1-e^{-\pi^{2} s^{2}}}{\operatorname{det} \Delta} .
$$

A direct computation gives

$$
\operatorname{det} \Delta=\left(1-e^{-s^{2}}\right)^{2}-s^{4} e^{-s^{2}}
$$

and

$$
\Omega=\left(\begin{array}{cc}
A & B \\
B & A
\end{array}\right)
$$

where

$$
A=\frac{1-e^{-s^{2}}-s^{2} e^{-s^{2}}}{\operatorname{det} \Delta}, \quad B=\frac{e^{-s^{2} / 2}\left(e^{-s^{2}}+s^{2}-1\right)}{\operatorname{det} \Delta} .
$$

Since

$$
\int_{-\infty}^{\infty} \int_{-\infty}^{\infty}\left|y_{1} y_{2}\right| e^{-\frac{1}{2}\left(A y_{1}^{2}+A y_{2}^{2}+2 B y_{1} y_{2}\right)} d y_{1} d y_{2}=\frac{4}{\operatorname{det} \Omega}\left(1+\frac{\delta}{\sqrt{1-\delta^{2}}} \arcsin \delta\right),
$$

where $\delta=B / A$ (see Appendix A), we obtain from (5.25) that

$$
\begin{aligned}
k_{2}\left(s_{1}, s_{2}\right) & =\frac{1}{(\operatorname{det} \Delta)^{1 / 2} \operatorname{det} \Omega}\left(1+\frac{\delta}{\sqrt{1-\delta^{2}}} \arcsin \delta\right) \\
& =\frac{(\operatorname{det} \Delta)^{1 / 2}}{1-e^{-s^{2}}}\left(1+\frac{\delta}{\sqrt{1-\delta^{2}}} \arcsin \delta\right) \\
& =\frac{\left[\left(1-e^{-s^{2}}\right)^{2}-s^{4} e^{-s^{2}}\right]^{1 / 2}}{1-e^{-s^{2}}}\left(1+\frac{\delta}{\sqrt{1-\delta^{2}}} \arcsin \delta\right),
\end{aligned}
$$

where $s=\pi\left(s_{1}-s_{2}\right)$ and

$$
\delta=\frac{e^{-s^{2} / 2}\left(e^{-s^{2}}+s^{2}-1\right)}{1-e^{-s^{2}}-s^{2} e^{-s^{2}}}
$$

It is worth to note that Hannay [Han] has calculated the limit two-point correlation function of zeros for the complex random $\mathrm{SU}(2)$ polynomial, and our calculation of (5.35) is very similar to the one of Hannay.

As $s \rightarrow 0$,

$$
\operatorname{det} \Delta=\frac{s^{8}}{12}+O\left(s^{10}\right), \quad \delta=1-\frac{s^{2}}{6}+O\left(s^{4}\right),
$$


which implies that

$$
k_{2}\left(s_{1}, s_{2}\right)=\frac{\pi^{2}\left|s_{1}-s_{2}\right|}{4}+O\left(\left|s_{1}-s_{2}\right|^{2}\right), \quad s_{1}-s_{2} \rightarrow 0 .
$$

As $s \rightarrow \infty$,

$$
\operatorname{det} \Delta=1-s^{4} e^{-s^{2}}+O\left(e^{-s^{2}}\right), \quad \delta=-s^{2} e^{-s^{2} / 2}+O\left(e^{-s^{2} / 2}\right),
$$

which implies that

$$
k_{2}\left(s_{1}, s_{2}\right)=1+\frac{\pi^{4}\left(s_{1}-s_{2}\right)^{4} e^{-\pi^{2}\left(s_{1}-s_{2}\right)^{2}}}{2}+O\left(\left(s_{1}-s_{2}\right)^{2} e^{-\pi^{2}\left(s_{1}-s_{2}\right)^{2}}\right), \quad\left|s_{1}-s_{2}\right| \rightarrow \infty .
$$

Thus we have proved the following theorem.

Theorem 5.1. Let $\left\{\tau_{j}\right\}$ be zeros of a random SO(2) polynomial $f_{n}(t)$ of degree $n$, and

$$
\zeta_{j}=\frac{\sqrt{n} \arctan \tau_{j}}{\pi} .
$$

be the straightened zeros. Then the limit m-point correlation function $k_{m}\left(s_{1}, \ldots, s_{m}\right)$ of $\left\{\zeta_{j}\right\}$ is given by the formula (5.29) where $\Delta$ is a $(2 m) \times(2 m)$ symmetric matrix which consists of $2 \times 2$ blocks $\Delta_{i j}$ defined in (5.26), and $\Omega=\left(\Delta^{-1}\right)$ even is the $m \times m$ matrix of the elements of $\Delta^{-1}$ with even indices. The 2-point correlation function is given by the formula (5.35), and its asymptotics as $s_{1}-s_{2} \rightarrow 0$ and $\left|s_{1}-s_{2}\right| \rightarrow \infty$ are given in (5.37) and (5.38), respectively.

The graph of $k_{2}(0, s)$ is shown in Fig.2.

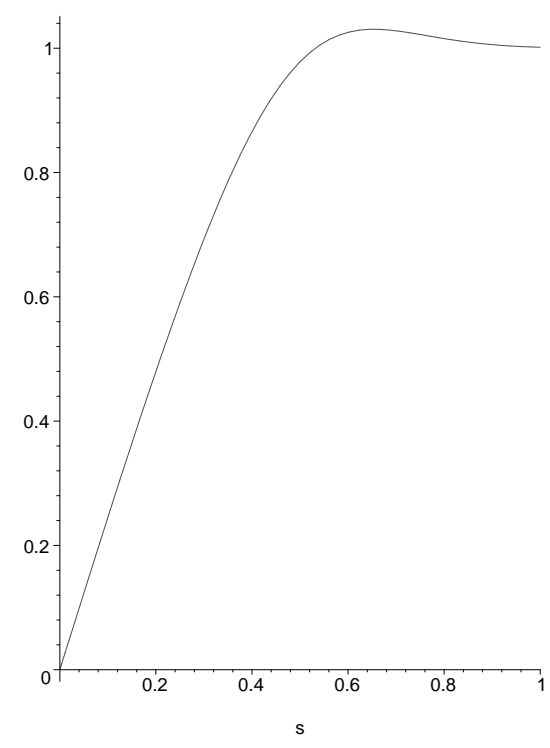

Fig 2: The two-point correlation function of straightened zeros of the $\mathrm{SO}(2)$ random polynomial. 


\section{Variance of the number of real zeros of the $\mathrm{SO}(2)$ random polynomial}

Here we calculate the variance of the random variable $\xi_{n}(a, b)$ in the case when $f_{n}(t)$ is the $\mathrm{SO}(2)$ random polynomial. By definition,

$$
\operatorname{Var} \xi_{n}(a, b)=\mathrm{E} \xi_{n}^{2}(a, b)-\left(\mathrm{E} \xi_{n}(a, b)\right)^{2} .
$$

By (2.17),

$$
\mathrm{E} \xi_{n}^{2}(a, b)=\int_{a}^{b} p_{n}(t) d t+\int_{a}^{b} \int_{a}^{b} K_{n 2}\left(t_{1}, t_{2}\right) d t_{1} d t_{2} .
$$

Since

$$
\left(\mathrm{E} \xi_{n}(a, b)\right)^{2}=\int_{a}^{b} p_{n}\left(t_{1}\right) d t_{1} \int_{a}^{b} p_{n}\left(t_{2}\right) d t_{2}
$$

we obtain that

$$
\begin{aligned}
\operatorname{Var} \xi_{n}(a, b) & =\int_{a}^{b} p_{n}(t) d t+\int_{a}^{b} \int_{a}^{b}\left[K_{n 2}\left(t_{1}, t_{2}\right)-p_{n}\left(t_{1}\right) p_{n}\left(t_{2}\right)\right] d t_{1} d t_{2} \\
& =\int_{a}^{b} p_{n}(t) d t+\int_{a}^{b} \int_{a}^{b}\left[\frac{K_{n 2}\left(t_{1}, t_{2}\right)}{p_{n}\left(t_{1}\right) p_{n}\left(t_{2}\right)}-1\right] p_{n}\left(t_{1}\right) p_{n}\left(t_{2}\right) d t_{1} d t_{2}
\end{aligned}
$$

When $t_{1}$ and $t_{2}$ are separated, the difference

$$
\frac{K_{n 2}\left(t_{1}, t_{2}\right)}{p_{n}\left(t_{1}\right) p_{n}\left(t_{2}\right)}-1
$$

is exponentially small, hence the main contribution to the last integral comes from close $t_{1}, t_{2}$. Let us put

$$
t_{1}=t, \quad t_{2}=t+\frac{s}{p_{n}(t)}
$$

Then

$$
\int_{a}^{b} \int_{a}^{b}\left[\frac{K_{n 2}\left(t_{1}, t_{2}\right)}{p_{n}\left(t_{1}\right) p_{n}\left(t_{2}\right)}-1\right] p_{n}\left(t_{1}\right) p_{n}\left(t_{2}\right) d t_{1} d t_{2} \sim \int_{a}^{b} p_{n}(t) d t \int_{-\infty}^{\infty}\left(k_{2}(0, s)-1\right) d s
$$

hence

$$
\operatorname{Var} \xi_{n}(a, b) \sim \int_{a}^{b} p_{n}(t) d t\left[1-\int_{-\infty}^{\infty}\left(1-k_{2}(0, s)\right) d s\right] .
$$

Thus

$$
\operatorname{Var} \xi_{n}(a, b) \sim C \sqrt{n} \int_{a}^{b} \frac{d t}{\pi\left(1+t^{2}\right)},
$$

where

$$
C=1-\int_{-\infty}^{\infty}\left(k_{2}(0, s)-1\right) d s
$$

and $k_{2}\left(s_{1}, s_{2}\right)$ is the two-point correlation fumction given in (5.35). In particular,

$$
\operatorname{Var} \xi_{n}(-\infty, \infty) \sim C \sqrt{n} .
$$

Numerical value of $C$ is

$$
C=0.5717310486902 \ldots
$$




\section{Appendix A. Calculation of an Integral}

In this appendix we show that

$$
\int_{-\infty}^{\infty} \int_{-\infty}^{\infty}\left|y_{1} y_{2}\right| e^{-\frac{1}{2}\left(A y_{1}^{2}+C y_{2}^{2}+2 B y_{1} y_{2}\right)} d y_{1} d y_{2}=\frac{4}{A C-B^{2}}\left(1+\frac{\delta}{\sqrt{1-\delta^{2}}} \arcsin \delta\right),
$$

where $\delta=B / \sqrt{A C}$. By a change of variables we can reduce the integral (A.1) to

$$
I=\int_{-\infty}^{\infty} \int_{-\infty}^{\infty}\left|y_{1} y_{2}\right| e^{-\frac{1}{2}\left(y_{1}^{2}+y_{2}^{2}+2 \delta y_{1} y_{2}\right)} d y_{1} d y_{2}
$$

Changing then

$$
\begin{aligned}
& y_{1}=\frac{1}{\sqrt{2}}\left(x_{1}+x_{2}\right), \\
& y_{2}=\frac{1}{\sqrt{2}}\left(x_{1}-x_{2}\right),
\end{aligned}
$$

we obtain that

$$
I=\frac{1}{2} \int_{-\infty}^{\infty} \int_{-\infty}^{\infty}\left|x_{1}^{2}-x_{2}^{2}\right| e^{-\frac{1}{2}\left[(1+\delta) x_{1}^{2}+(1-\delta) x_{2}^{2}\right]} d x_{1} d x_{2}
$$

Let

$$
\begin{aligned}
& x_{1}=\frac{r}{\sqrt{1+\delta}} \cos \theta, \\
& x_{2}=\frac{r}{\sqrt{1-\delta}} \sin \theta .
\end{aligned}
$$

Then

$$
\begin{aligned}
I & =\frac{1}{2\left(1-\delta^{2}\right)^{1 / 2}} \int_{0}^{\infty} r^{3} e^{-\frac{r^{2}}{2}} d r \int_{0}^{2 \pi}\left|\frac{\cos ^{2} \theta}{1+\delta}-\frac{\sin ^{2} \theta}{1-\delta}\right| d \theta \\
& =\frac{1}{\left(1-\delta^{2}\right)^{3 / 2}} \int_{0}^{2 \pi}\left|(1-\delta) \cos ^{2} \theta-(1+\delta) \sin ^{2} \theta\right| d \theta \\
& =\frac{1}{\left(1-\delta^{2}\right)^{3 / 2}} \int_{0}^{2 \pi}|\delta-\cos 2 \theta| d \theta
\end{aligned}
$$

Evaluating the last integral we obtain that

$$
I=\frac{4}{\left(1-\delta^{2}\right)^{3 / 2}}\left(\sqrt{1-\delta^{2}}+\delta \arcsin \delta\right)
$$

hence (A.1) is proved. 


\section{Appendix B. Positivity of the Covariance Matrix}

Assume that $f_{n}(t)=c_{0}+c_{1} t+\cdots+c_{n} t^{n}$ is a polynomial with independent random coefficients such that $\mathrm{E} c_{k}=0$ and $0<\mathrm{E} c_{k}^{2}<\infty$. We show in this appendix that the covariance matrix of the random vector

$$
F_{n}=\left(f_{n}\left(t_{1}\right), f_{n}^{\prime}\left(t_{1}\right), \ldots, f_{n}\left(t_{m}\right), f_{n}^{\prime}\left(t_{m}\right)\right)
$$

is positive, if $t_{1}, \ldots, t_{m}$ are pairwise different and $n \geq 2 m-1$. Consider the real-valued quadratic form associated with the covariance matrix,

$$
\begin{aligned}
Q(\alpha, \beta) & =\sum_{j, k}\left[\alpha_{j} \alpha_{k} \mathrm{E} f_{n}\left(t_{j}\right) f_{n}\left(t_{k}\right)+\alpha_{j} \beta_{k} \mathrm{E} f_{n}\left(t_{j}\right) f_{n}^{\prime}\left(t_{k}\right)\right. \\
& \left.+\beta_{j} \alpha_{k} \mathrm{E} f_{n}^{\prime}\left(t_{j}\right) f_{n}\left(t_{k}\right)+\beta_{j} \beta_{k} \mathrm{E} f_{n}^{\prime}\left(t_{j}\right) f_{n}\left(t_{k}\right)\right] \\
& =\mathrm{E}\left|\sum_{j}\left[\alpha_{j} f_{n}\left(t_{j}\right)+\beta_{j} f_{n}^{\prime}\left(t_{j}\right)\right]\right|^{2} \\
& =\sum_{k=0}^{n} \sigma_{k}^{2}\left[\sum_{j=1}^{m}\left(\alpha_{j} t_{j}^{k}+\beta_{j} k t_{j}^{k-1}\right)\right]^{2}
\end{aligned}
$$

The generalized Vandermonde matrix

$$
\left(t_{j}^{k}, k t_{j}^{k-1}\right)_{j=1, \ldots, m ; k=0, \ldots, 2 m-1}
$$

is nondegenerate, hence

$$
\sum_{k=0}^{n} \sigma_{k}^{2}\left[\sum_{j=1}^{m}\left(\alpha_{j} t_{j}^{k}+\beta_{j} k t_{j}^{k-1}\right)\right]^{2}>0
$$

provided that not all $\alpha_{j}, \beta_{j}$ are zero. This proves that the quadratic form $Q(\alpha, \beta)$ is positive, hence the covariance matrix of the random vector (B.1) is positive as well.

The proof remains valid if $c_{k}$ are, in general, dependent random variables with positive covariance matrix $\left(V_{k l}=\mathrm{E} c_{k} c_{l}\right)_{k, l=0, \ldots, n}$. In this case

$$
Q(\alpha, \beta)=\sum_{k, l=0}^{n} V_{k l} d_{k} d_{l}>0, \quad d_{k}=\sum_{j=1}^{m}\left(\alpha_{j} t_{j}^{k}+\beta_{j} k t_{j}^{k-1}\right),
$$

provided that $n \geq 2 m-1$ and not all $\alpha_{j}, \beta_{j}$ are zero. 


\section{Appendix C. Proof of the Inequality $\Delta>0$}

Let $\Delta=\left(\Delta_{j k}\right)_{j, k=1, \ldots, m}$ where

$$
\Delta_{j k}=e^{-\left(s_{j}-s_{k}\right)^{2} / 2}\left(\begin{array}{cc}
1 & \left(s_{j}-s_{k}\right) \\
\left(s_{k}-s_{j}\right) & 1-\left(s_{j}-s_{k}\right)^{2}
\end{array}\right)
$$

We prove in this appendix that $\Delta>0$. Consider the complex-valued quadratic form associated with $\Delta$,

$$
Q(\alpha, \beta)=\sum_{j, k} e^{-\left(s_{j}-s_{k}\right)^{2} / 2}\left[\alpha_{j} \bar{\alpha}_{k}+\left(s_{j}-s_{k}\right) \alpha_{j} \bar{\beta}_{k}+\left(s_{k}-s_{j}\right) \beta_{j} \bar{\alpha}_{k}+\left[1-\left(s_{j}-s_{k}\right)^{2}\right] \beta_{j} \bar{\beta}_{k}\right] .
$$

Using the formulae

$$
\begin{aligned}
e^{-\left(s_{j}-s_{k}\right)^{2} / 2} & =\frac{1}{\sqrt{2 \pi}} \int_{-\infty}^{\infty} e^{i\left(s_{j}-s_{k}\right) x} e^{-x^{2} / 2} d x, \\
\left(s_{j}-s_{k}\right) e^{-\left(s_{j}-s_{k}\right)^{2} / 2} & =\frac{1}{\sqrt{2 \pi}} \int_{-\infty}^{\infty} e^{i\left(s_{j}-s_{k}\right) x}(-i x) e^{-x^{2} / 2} d x, \\
{\left[1-\left(s_{j}-s_{k}\right)^{2}\right] e^{-\left(s_{j}-s_{k}\right)^{2} / 2} } & =\frac{1}{\sqrt{2 \pi}} \int_{-\infty}^{\infty} e^{i\left(s_{j}-s_{k}\right) x} x^{2} e^{-x^{2} / 2} d x,
\end{aligned}
$$

we can rewrite $Q$ as

$$
\begin{aligned}
Q(\alpha, \beta) & =\frac{1}{\sqrt{2 \pi}} \int_{-\infty}^{\infty} d x e^{-x^{2} / 2} \sum_{j, k}\left[\alpha_{j} \bar{\alpha}_{k} e^{i\left(s_{j}-s_{k}\right) x}+\alpha_{j} \bar{\beta}_{k} e^{i\left(s_{j}-s_{k}\right) x}(-i x)\right. \\
& \left.+\beta_{j} \bar{\alpha}_{k} e^{i\left(s_{j}-s_{k}\right) x}(i x)+\beta_{j} \bar{\beta}_{k} e^{i\left(s_{j}-s_{k}\right) x} x^{2}\right] .
\end{aligned}
$$

Define

$$
f(x)=\sum_{j=1}^{m} \alpha_{j} e^{i s_{j} x}, \quad g(x)=\sum_{j=1}^{m} \beta_{j} e^{i s_{j} x} .
$$

Then

$$
Q(\alpha, \beta)=\frac{1}{\sqrt{2 \pi}} \int_{-\infty}^{\infty}|f(x)+i x g(x)|^{2} e^{-x^{2} / 2} d x
$$

The function $f(x)+i x g(x)$ is not identically zero provided that not all $\alpha_{j}, \beta_{j}$ are 0 . Indeed, for every test function $\varphi(x)$,

$$
\int_{-\infty}^{\infty}(f(x)+i x g(x)) \varphi(x) d x=\sum_{j=1}^{m}\left(\alpha_{j} \tilde{\varphi}\left(s_{j}\right)+\beta_{j} \tilde{\varphi}^{\prime}\left(s_{j}\right)\right),
$$

where $\tilde{\varphi}(s)$ is the Fourier transform of $\varphi(x)$. We can localize the function $\tilde{\varphi}(s)$ near $s_{j}$ and make the last sum nonzero. This proves that $f(x)+i x g(x)$ is not identically zero, and hence $Q(\alpha, \beta)>0$. Hence $\Delta>0$. 
Acknowledgement. This work was supported in part by the National Science Foundation, Grant No. DMS-9623214, and this support is gratefully acknowledged.

\section{References}

[BS] A.T. Bharucha-Reid and M. Sambandham, Random polynomials, Academic Press, New York, 1986.

[BP] A. Bloch and G. Polya, On the roots of certain algebraic equations, Proc. London Math. Soc. (3) 33 (1932), 102-114.

[BBL1] E. Bogomolny, O. Bohigas and P. Leboeuf, Distribution of roots of random polynomials, Phys. Rev. Lett. 68 (1992), 2726-2729.

[BBL2] E. Bogomolny, O. Bohigas and P. Leboeuf, Quantum chaotic dynamics and random polynomials. Preprint, 1996.

[Dys] F. J. Dyson, Correlation between eigenvalues of a random matrix, Commun. Math. Phys. 19 (1970), 235-250.

[EK] A. Edelman and E. Kostlan, How many zeros of a random polynomial are real? Bull. Amer. Math. Soc., 32 (1995) 1-37.

[EO] P. Erdös and A. Offord, On the number of real roots of random algebraic equation, Proc. London Math. Soc. 6 (1956), 139-160.

[ET] P. Erdös and P. Turán, On the distribution of roots of polynomials, Ann. Math. 51 (1950), 105-119.

[Han] J. H. Hannay, Chaotic analytic zero points: exact statistics for those of a random spin state, J. Phys. A: Math. Gen. 29 (1996), 101-105.

[IM] I. A. Ibragimov and N. B. Maslova, On the average number of real zeros of random polynomials. I, II, Teor. Veroyatn. Primen. 16 (1971), 229-248, 495-503.

[K1] M. Kac, On the average number of real roots of a random algebraic equation, Bull. Amer. Math. Soc. 49 (1943), 314-320.

[K2] M. Kac, On the average number of real roots of a random algebraic equation. II, Proc. London Math. Soc. 50 (1948), 390-408. 
[K3] M. Kac, Probability and related topics in physical sciences. Wiley (Interscience), New York, 1959.

[Kos] E. Kostlan, On the distribution of roots of random polynomials, From Topology to Computation: Proceedings of the Smalefest (M. W. Hirsh, J. E. Marsden, and M. Shub, eds.), Springer-Verlag, New York, 1993, pp. 419-431.

[Leb1] P. Leboeuf, Phase space approach to quantum dynamics, J. Phys. A: Math. Gen. 24 (1991), 4575.

[Leb2] P. Leboeuf, Statistical theory of chaotic wavefunctions: a model in terms of random analytic functions, Preprint, 1996.

[LS] P. Leboeuf and P. Shukla, Universal Fluctuations of zeros of Chaotic Wavefunctions, Preprint, 1996.

[LO] J. Littlewood and A. Offord, On the number of real roots of random algebraic equation. I,II,III, J. London Math. Soc. 13 (1938), 288-295; Proc. Cambr. Phil. Soc. 35 (1939), 133-148; Math. Sborn. 12 (1943), 277-286.

[LS] B. F. Logan and L. A. Shepp, Real zeros of a random polynomial. I, II, Proc. London Math. Soc. 18 (1968), 29-35, 308-314.

[Maj] E. Majorana, Nuovo Cimento 9 (1932), 43-50.

[Mas1] N. B. Maslova, On the variance of the number of real roots of random polynomials, Teor. Veroyatn. Primen. 19 (1974), 36-51.

[Mas2] N. B. Maslova, On the distribution of the number of real roots of random polynomials, Teor. Veroyatn. Primen. 19 (1974), 488-500.

[Meh] M. L. Mehta, Random matrices, Academic Press,New York, 1991.

[M-A] G. Mezincescu, D. Bessis, J. Fournier, G. Mantica, and F. D. Aaron, Distribution of roots of random real polynomials. Preprint, 1996.

[SS] M. Shub and S. Smale, Complexity of Bezout's Theorem II: Volumes and Probabilities, Computational Algebraic Geometry (F. Eysette and A. Calligo, eds.), Progr. Math., vol. 109, Birkhauser, Boston, 1993, pp. 267-285.

[Ste] D. Stevens, Expected number of real zeros of polynomials, Notices Amer. Math. Soc. 13 (1966), 79. 